\title{
Surge Voltage Breakdown of Air in a Nonuniform Field
}

\author{
J. H. Park and H. N. Cones
}

\begin{abstract}
The discharge and breakdown phenomena in air when a surge voltage is applied to sphere-plane electrodes were investigated. A steeply rising surge of 145 kilovolts peak value was applied to the plane placed 86 centimeters above the laboratory floor. A 1.6centimeter diameter sphere, mounted an adjustable distance below the plane, was connected to ground through the surge impedance of a coaxial cable. Experimental data consisted of oscillograms of the current to the sphere and pictures of the discharge between the electrodes. A method for chopping the applied voltage surge at an accurately controllable time was used to study the discharge at gap spacings for which a full-wave applied surge would cause breakdown.

Complete breakdown did not occur for gap spacings greater than 28 centimeters, sphere positive, or 20 centimeters, sphere negative. But at gap spacings up to 56 centimeters, sphere positive, and 46 centimeters, sphere negative, discharge streamers (corona) did develop from the sphere and they were accompanied by short pulses of current to the sphere. At gap spacings near those giving breakdown, these initial streamers span the gap without yielding breakdown. Measured speeds of formation of these initial streamers were found to be 500 centimeters per microsecond for the sphere negative and 800 centimeters per microsecond for the sphere positive.

For shorter gap spacings the discharge leading to breadown is illustrated by the data obtained with the applied voltage chopped. Bright conducting channels develop between the sphere and plane. At the same time the current to the sphere starts to rise again fairly slowly at first and then at an increasing rate up to breakdown. The mechanism leading to breakdown is not the same for the sphere negative as for the sphere positive, and it is different for very short gaps which correspond to high overvoltage. An attempt is made to explain how the initial streamers form and how the channels leading to breakdown develop.
\end{abstract}

\section{Introduction}

Electrical breakdown in air has been extensively studied by a large number of investigators and the many papers and books already published on this subject have contributed greatly toward an understanding of the mechanism of breakdown. The parameters most likely to affect breakdown mechanism are (1) gas pressure, (2) size, shape, and separation of electrodes, and (3) waveform of the applied voltage. No single simple theory can be expected to explain breakdown of a gas under all possible experimental conditions. The original Townsend theory with some modifications $[1,2]^{1}$ is well established as giving the breakdown mechanism of gases at pressures much below atmospheric, and in some recent theoretical analyses $[3,4]$ the Townsend theory is extended to include breakdown at amospheric pressures. However, the streamer theory of Loeb and Meek [5] or some modification of it appears most likely to account for the short formative time lags experienced in uniform field breakdown at atmospheric pressure. Breakdown studies at high frequencies appear to require an entirely different theory to adequately explain the results, and a theory that seems adequate has been proposed $[6,7,8]$.

The theories referred to above are based mainly on uniform field breakdown, largely because this has seemed the simpler case. For the same reason, most of the experimental data already published have been for gaps giving a uniform or nearly uniform

1 Figures in brackets indicate the literature references at the end of this paper field. At the higher voltages almost all of these data have been obtained with sphere gaps because such gaps are used as primary reference standards in the field of high voltage measurements. For the design of high voltage electrical equipment and in the development of methods for measuring steeply rising voltage surges, a knowledge of just how breakdown occurs when a voltage is suddenly applied to electrodes producing a nonuniform field is of primary importance. Results obtained by Norinder and others $[9,10,11,12,13]$ indicate that the mechanism of breakdown for nonuniform field gaps is quite different from that for uniform field gaps. The work described in this paper was undertaken in order to gain a better understanding of the mechanism of breakdown for nonuniform fields.

\section{Scope of the Experimental Work}

The present work is limited to discharge phenomena and breakdown in air, under the usual laboratory conditions of pressure and humidity, when voltage is suddenly applied and the electrode geometry is such that a nonuniform electric field is produced in the gap. The laboratory floor which has a grounded metal grid imbedded in its surface was used as a semi-infinite ground plane. The upper or highvoltage electrode consisted of a large circular plane, $84 \mathrm{~cm}$ (33 in.) in diameter, made of an aluminum alloy, placed $86.4 \mathrm{~cm}$ (34 in.) above and parallel to the ground plane. The ground electrode was a 1.6-cm ( $(5 / 8-i n$.$) diameter sphere mounted at the end$ of a conductor centered inside a $0.635-\mathrm{cm}$ ( $1 / 4-\mathrm{in}$.) 
diameter metal tube and insulated from it. The sphere was located at an adjustable distance $L$ beneath the center of the high-voltage plane electrode as indicated schematically in figure 1. A metalcovered trench in the floor permitted the sphere mounting tube to extend into the trench through a hole in the cover. The tube was grounded at the metal trench cover, and in the trench below its cover, the tube and its center conductor were connected to a coaxial cable. This cable ran in the trench to a cathode ray oscillograph $(\mathrm{CRO})$ where it was terminated by its characteristic impedance of $50 \mathrm{ohms}$. Thus the sphere was "in effect" connected directly to ground through a 50-ohm resistance and any current from the sphere to the high-voltage plane electrode could be measured by the IR drop it produced at the oscillograph. In addition to permitting an accurate measurement of prebreakdown current between the sphere and the plane when voltage is suddenly applied to the plane, this electrode arrangement ${ }^{2}$ also makes it possible to compute the initial electric field at various distances from the sphere toward the plane (see the appendix for a summary of such computations). The voltage gradient is of course maximum at the surface of the sphere, allow-. ing significant polarity effects to be studied.

The main purpose of the present investigation was to obtain data indicating the mechanism of breakdown. A study of discharge phenomena could be approached in two ways: (1) by holding the peak voltage of the applied surge constant and changing the gap spacing or (2) by holding the gap spacing constant and changing the peak voltage. Since it is experimentally quite troublesome to maintain the same rise time for various values of peak voltage, method (1) above was used. Voltage surges of fairly short duration and of the following wave shapes were applied to the gap: (1) a surge that rose to a peak value of $145 \mathrm{kv}$ in about $0.07 \mu \mathrm{sec}$ (as rapidly as possible with the setup used) and maintained this peak value fairly constant, gradually decreasing to half value in about $100 \mu \mathrm{sec}$ (designated as $0.07 \times 100$, 145 -kv surge); (2) a surge similar to (1) except that it rose to peak value in about $1 \mu \mathrm{sec}$ (designated as $1 \times 100,145-\mathrm{kv}$ surge); (3) surges similar to either (1) or (2) except that the voltage was chopped off at various times after reaching peak value (designated as $0.07 \times t_{c}, 145$-kv or $1 \times t_{c}, 145$-kv surge).

The sphere-plane electrode system was enclosed in a light-tight fiberboard box $3^{1 / 2}$ by 3 by $10 \mathrm{ft}$ $(106 \times 91 \times 305 \mathrm{~cm}) . \quad$ A $35-\mathrm{mm}$ camera with an $\mathrm{f} / 1.9$ coated lens was placed inside this enclosure at a distance of $30 \mathrm{in} .(76 \mathrm{~cm})$ from the gap for taking photographic records of the discharge between electrodes. This box also provided shielding for the test gap against any extraneous radiation. The effect of an increased ion and electron density at the gap before applying voltage was determined by placing a radioactive source near the sphere electrode. 2 The idea of putting high voltage on the plane electrode and grounding the
smaller electrode through a resistance so that current could be measured was also used by Saxe and Meek [13] in their recent work on this subject. The present used by Saxe and Meek [13] in their recent work on this subject. The present
paper was completed but not published at the time the Saxe and Meek paper was paper was com
publishied.

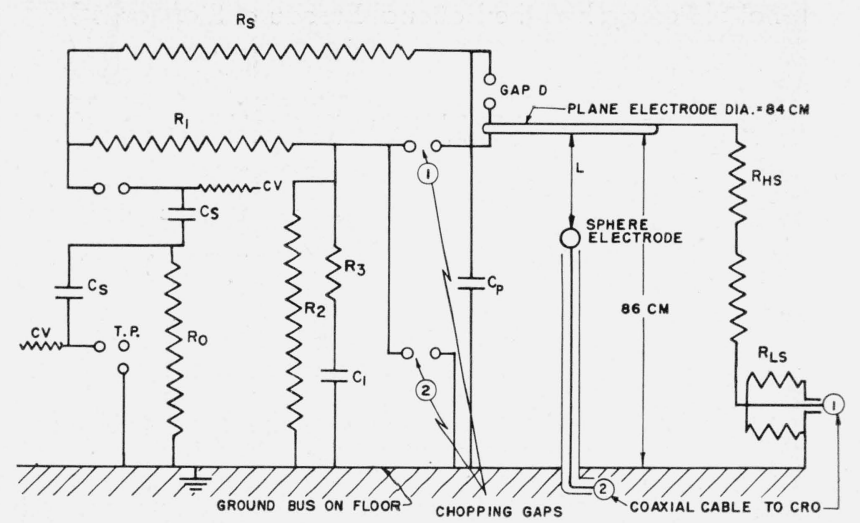

FIgure 1. Schematic diagram of sphere-plane electrodes showing the voltage supply and measuring circuits

At the two points marked "CV," one positive and the other negative, $100-\mathrm{kv}$ charging voltages were introduced. $\mathrm{C}_{\mathrm{s}}=1 / 3 \quad \mu \mathrm{f}, 100 \mathrm{kv}$ capacitor. $\mathrm{R}_{\mathrm{o}}=3,000$ ohms. $R_{1}=2,500$ ohms. $R_{8}=165$ ohms. $C_{p}=0.003 \mu$ f capacitor. $R_{3}=500$ ohms. $R_{2}=40,000$ ohms. $C_{1}=0.002 \mu$ capacitor. $R_{H s}$ and $R_{L s}$ form voltage divider.

\section{Experimental Setup and Procedure}

The arrangement of the sphere-plane electrodes is shown schematically in figure 1 . The divider for measuring the voltage applied to the plane is also shown. The high voltage part of this divider consists of a 1,000-ohm noninductive wire wound resistor, $R_{\mathrm{HS}}$, placed vertically to the floor at about $2 \mathrm{ft}$ from the edge of the plane electrode. The divider low side, $R_{\mathrm{LS}}$, is a specially constructed 5 -ohm noninductive resistor, arranged to produce minimum inductive effects from ground currents when it is connected to the coaxial cable to the CRO. A highvoltage cold-cathode oscillograph with beam intensification [14] was used to record the voltage waveform and to measure peak values. By connecting the CRO cable at (2) in figure 1 the same oscillograph was used to obtain a record of prebreakdown current. This arrangement of electrodes and measuring circuits was used throughout our experiments.

The circuit used for producing the surge voltage and for applying it to the plane-sphere gap is shown in figure 1 at the left. Capacitors, $\mathrm{C}_{\mathrm{s}}$, are 2 units from a 20-unit 2,000-kv surge voltage generator. They are each charged to $100 \mathrm{kv}$ by the full-wave kenotron rectifier of the surge voltage generator, one positive and the other negative with respect to ground. By applying a tripping pulse to the middle ball of the three-ball gap, the two capacitors, $\mathrm{C}_{\mathrm{s}}$, are connected in series to the discharge circuit. To obtain a full-wave voltage surge at the test gap, chopping gap (1) is set wide and (2) is shorted. Thus the two capacitors, $\mathrm{C}_{\mathrm{s}}$ in series, start discharging through $R_{1}$ in parallel with $R_{s}$ and $C_{p}$ in series. If gap $\mathrm{D}$ is shorted, the slowly rising voltage surge $(1 \times 100,145-\mathrm{kv}$ surge $)$ is applied to the planesphere gap. To obtain the fast-rising surge $(0.07 \times$ $100,145-\mathrm{kv}$ surge), gap D is set at 5 -cm spacing. This keeps the voltage off the plane-sphere gap during its slowly rising portion. The oscillograph records reproduced in figures 2 and 3 show the waveform of the steeply and slowly rising voltage surges, respectively. Details of the wave front are shown by the records obtained using a fast sweep. 
A

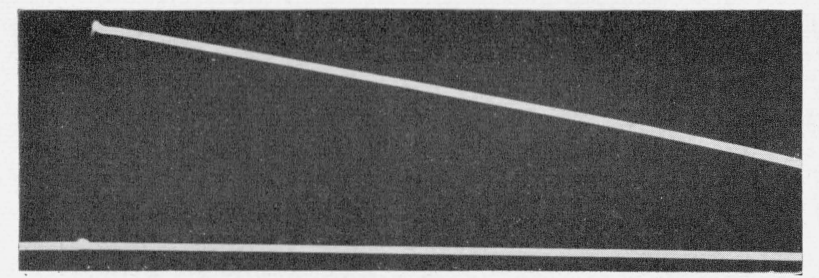

0

40

MICROSECONDS

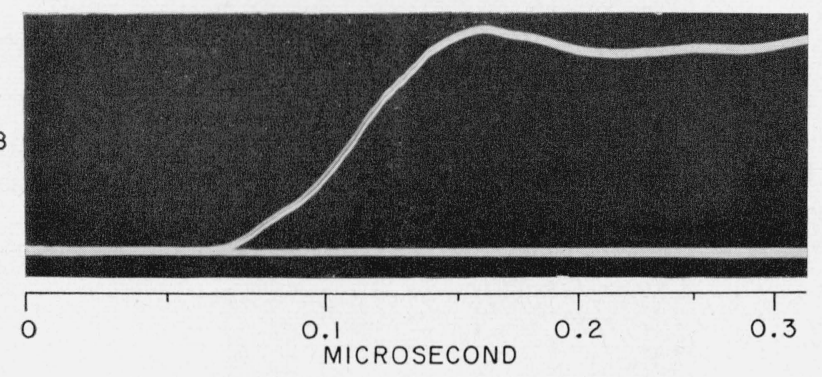

Figure 2. Oscillograms of $0.07 \times 100$, 145-kv voltage surge.

A, Slow sweep showing wave tail; B, fast sweep showing wave front.

The chopping gaps (1) and (2) together with $R_{1}$, $R_{2}, R_{3}$, and $C_{1}$ are used to chop the voltage applied to the plane electrode at a definite time after it is applied. This chopping circuit is similar to one described by G. H. Johnson [15]. As noted from the values of circuit constants given in figure 1, the time constant $R_{s} C_{p}$ is about $0.5 \mu$ sec and the time constant $\left(\mathrm{R}_{1}+\mathrm{R}_{3}\right) \mathrm{C}_{1}$ is about $5 \mu$ sec. Thus gap $\mathrm{D}$ fires very soon after the supply circuit is triggered and this puts voltage on the plane electrode. At the same time the voltage across chopping gap (2) begins to rise, and by adjusting gap (2) it can be set to fire from 1 to $6 \mu \mathrm{sec}$ after voltage is applied to the plane electrode. Before chopping gap (2) fires, the two balls of chopping gap (1) have voltages of the same polarity applied to them; thus gap (1) will not fire. But when gap (2) fires, the full voltage applied to the plane will appear across gap (1) and it will fire, thus chopping the supply voltage.

The procedure consisted, in general, of choosing a particular wave shape and polarity and then obtaining (1) CRO records of the sphere current and (2) photographs of the discharge between sphere and plane, for various values of gap spacing $L$. At values of $L$ for which the full voltage wave would cause complete breakdown between the sphere and plane, a series of pictures and CRO records of current were also taken with the applied voltages chopped at various times before complete breakdown.

Records of light output from the prebreakdown discharge were also obtained in some cases by using a photomultiplier tube and a high-speed oscilloscope with amplifier. The variations in light output followed the same pattern as the records of current to the sphere, but because this scheme is much more subject to extraneous pickup and calibration errors, the current records are considered to be more significant.
A
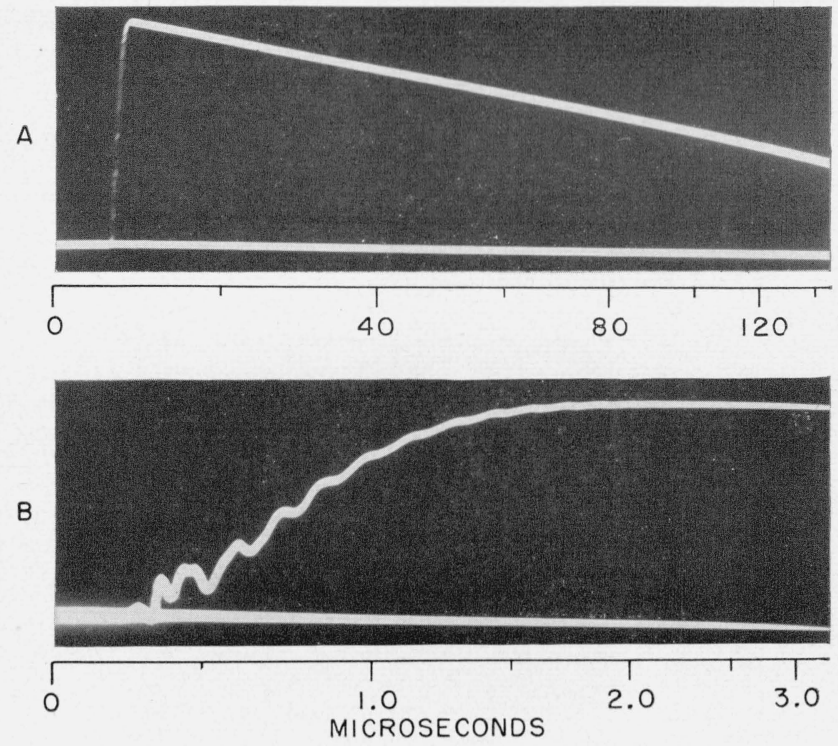

Figure 3. Oscillograms of $1 \times 100,145-k v$ voltage surge. A, Slow sweep showing wave tail. B, Fast sweep showing wave front.

\section{Experimental Results}

\subsection{Full-Wave 145-kv Surge}

Sets of data were obtained under four different conditions of applied voltage: (1) steeply rising surge $(.07 \times 100)$, sphere positive, (2) slowly rising surge $(1 \times 100)$, sphere positive, (3) steeply rising surge, sphere negative, and (4) slowly rising surge, sphere negative. For each condition of applied voltage, the test data included CRO records of the current to the sphere and corresponding photographs of the discharge, at various values of gap spacing $L$.

Typical results are shown in figures $4,5,6$, and 7 . The first small wiggles on the current records are caused by capacitance current to the sphere when voltage is suddenly applied. They are useful as reference points to show the exact time at which voltage is applied. Following the capacitance current wiggles there is a sudden rise in current followed by an approximately exponential decrease to zero. This will be called the "first discharge pip" because it occurs only on shots causing a visible discharge from the sphere. It does not constitute a complete breakdown of the gap. The first discharge pip is definitely not associated with capacitance pickup because on some shots it occurs when the rate of change of applied voltage is nearly zero as shown, for example, in figure $5 \mathrm{~A}$.

For gap spacings less than $28 \mathrm{~cm}$ (sphere positive) or $15 \mathrm{~cm}$ (sphere negative), complete breakdown between sphere and plane takes place on at least some of the shots. Complete breakdown is indicated on the current record (see figures $4 \mathrm{~F}, 5 \mathrm{E}, 6 \mathrm{~F}$, and 7C) by a second and roughly exponential increase in current to large values. This current rise follows the first discharge pip and will be referred to as the 

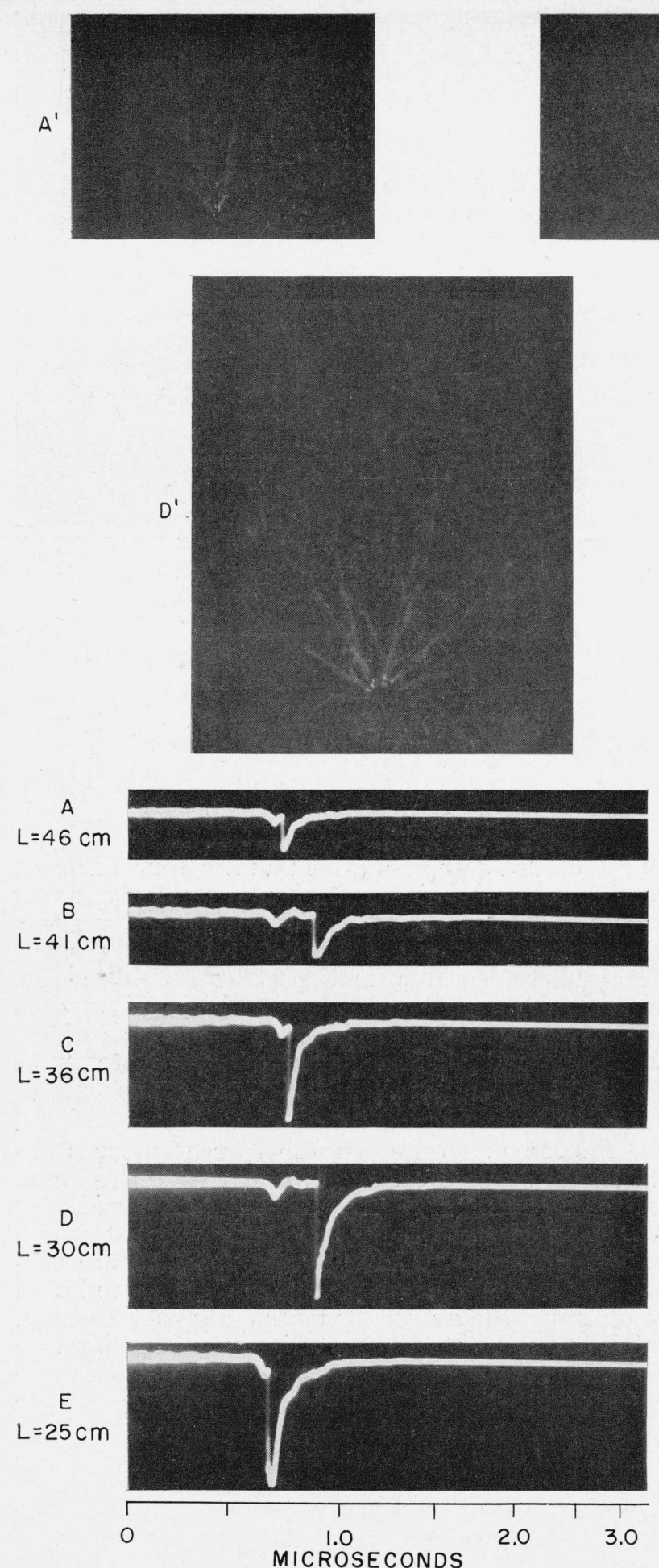

$B^{\prime}$
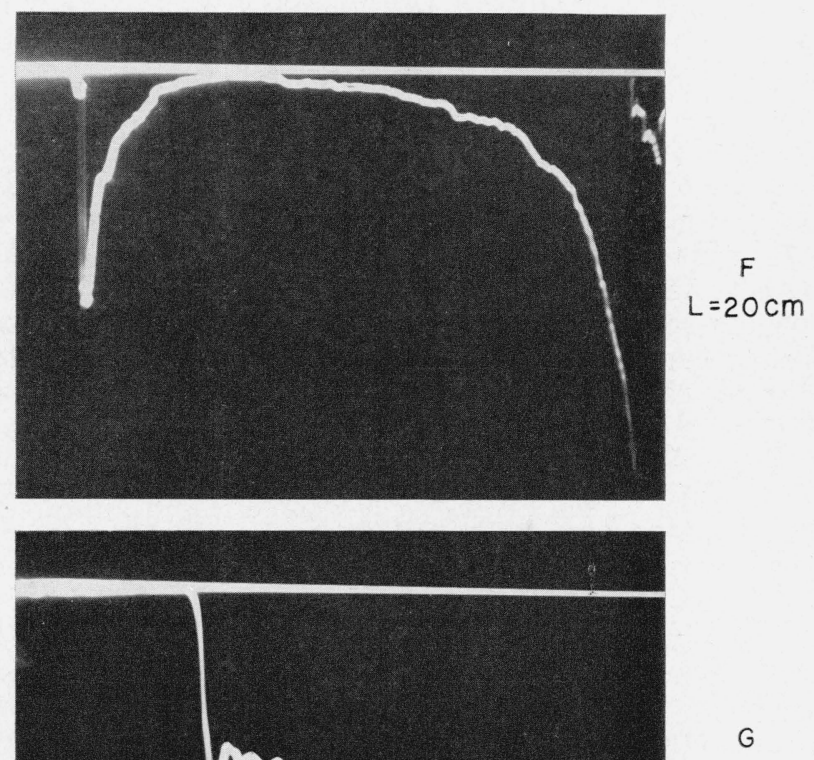

FIGURE 4. Oscillograms of prebreakdown current and discharge pictures taken on the same shots.

Sphere positive, $0.07 \times 100,145-\mathrm{kv}$ surge. Gap spacings $L$ as noted. $\mathrm{F}$ is current oscillogram for complete breakdown of gap. G is oscillogram of applied voltage. 


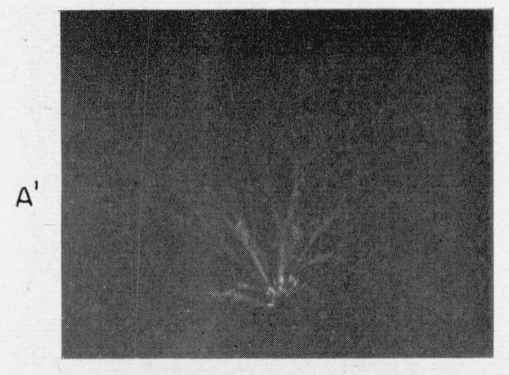

A

$L=41 \mathrm{~cm}$
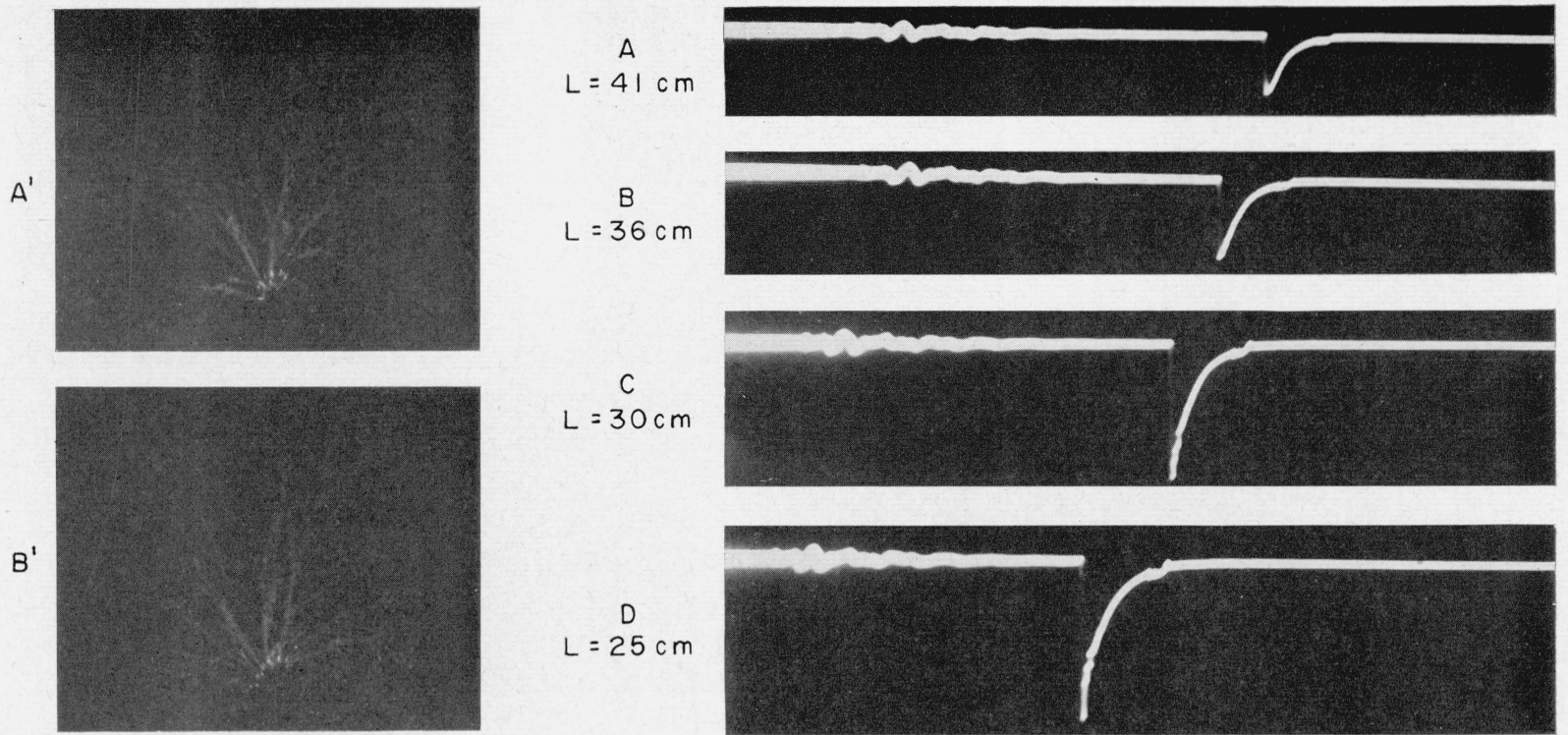

$B$
$L=36 \mathrm{~cm}$

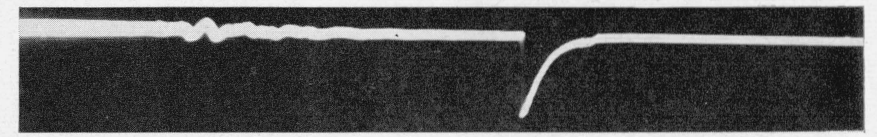

$C$
$=30 \mathrm{~cm}$
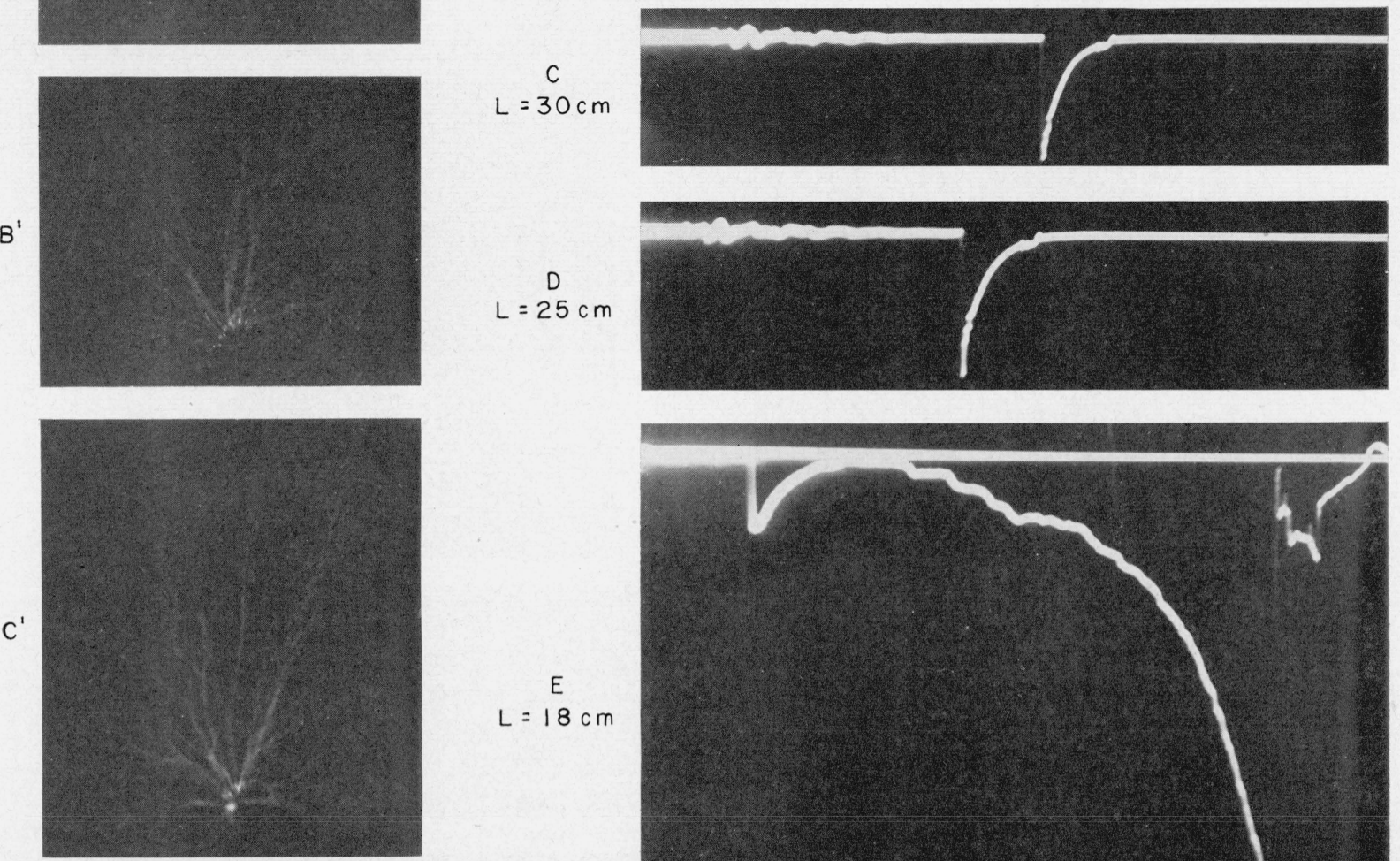

E

$L=18 \mathrm{~cm}$

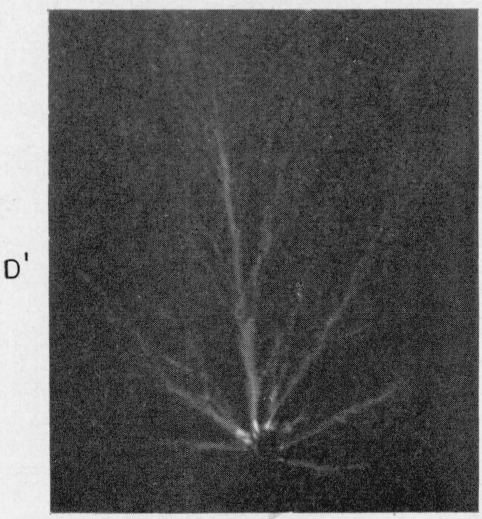

$L=25 \mathrm{~cm}$
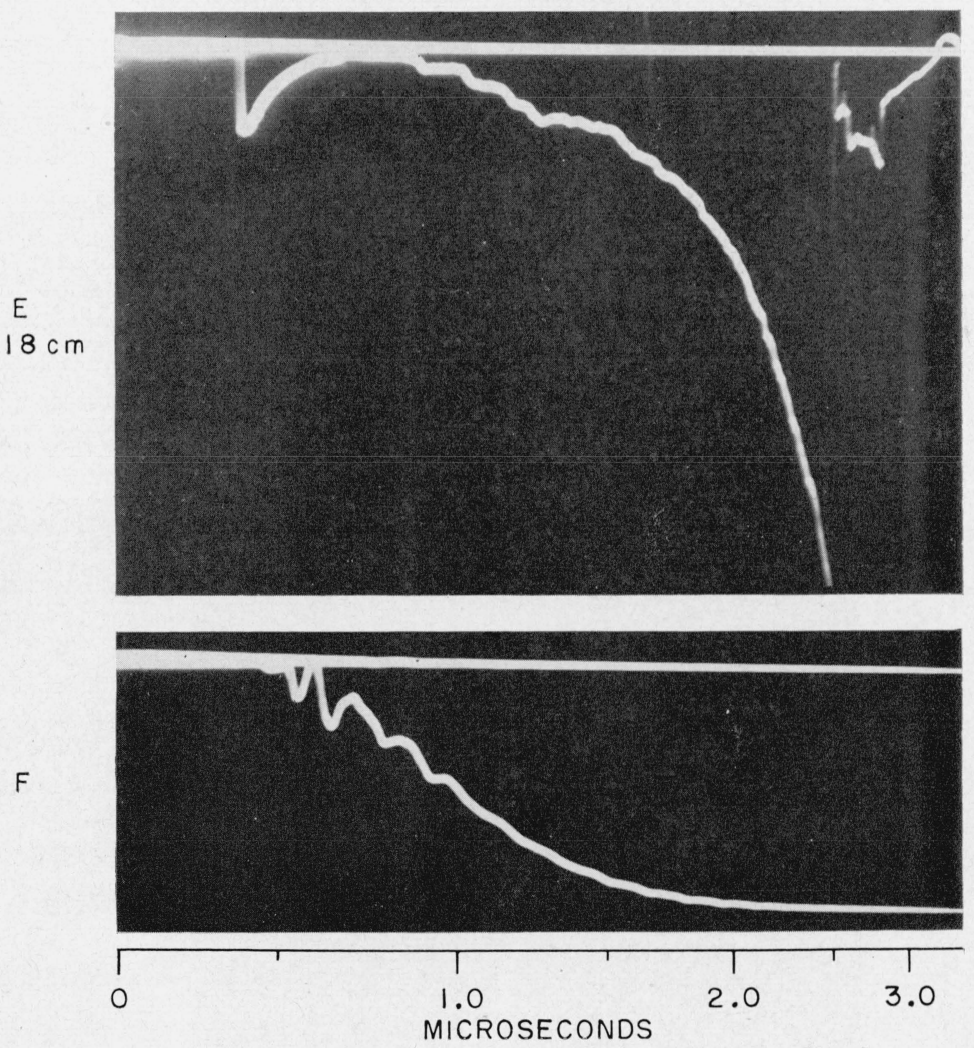

FIGURE 5. Oscillograms of prebreakdown current and discharge pictures taken on the same shots.

Sphere positive, $1 \times 100,145$-kv surge. Gap spacings $L$ as noted. E is current oscillogram for complete breakdown of gap. F is oscillogram of applied voltage. 

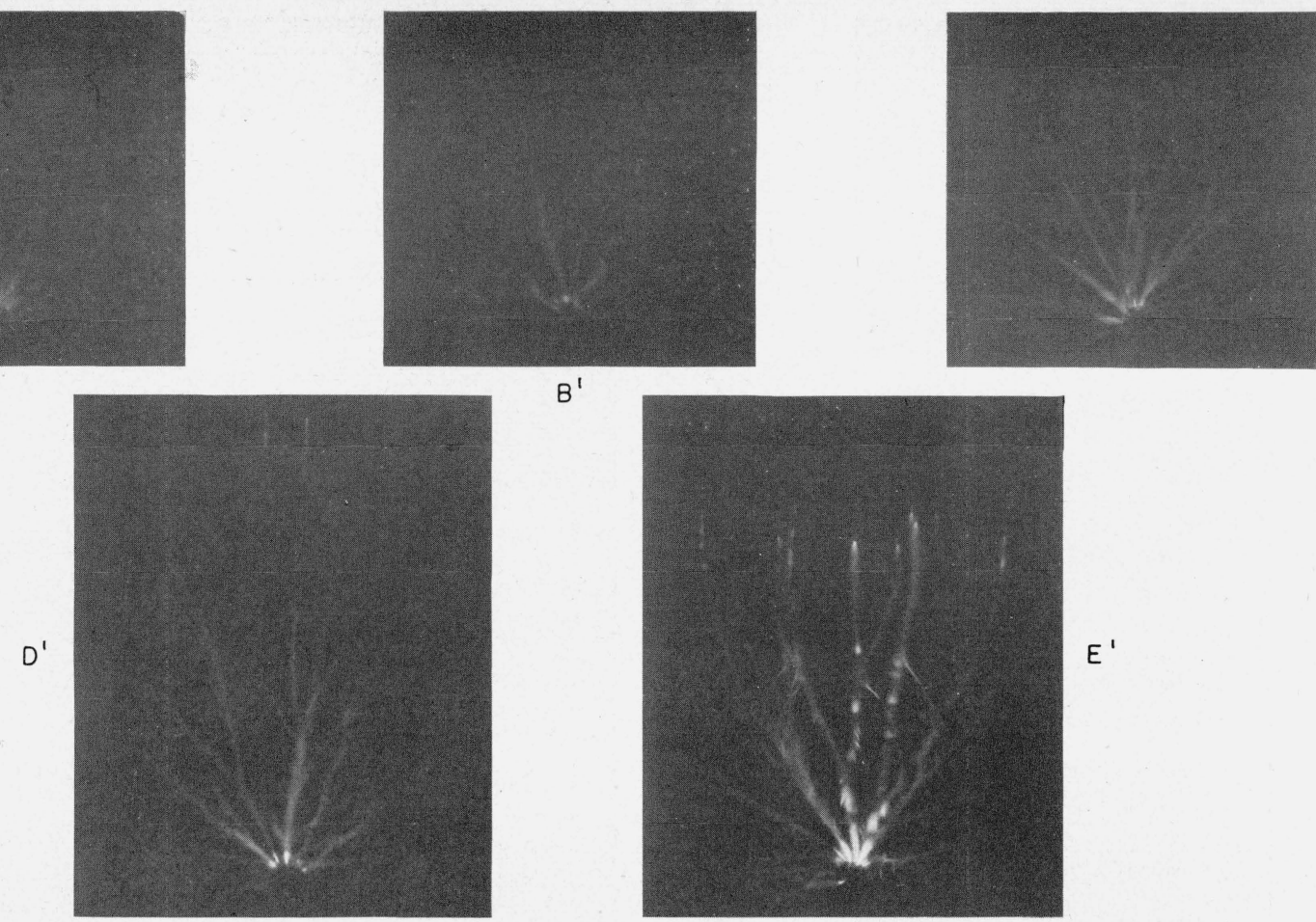

$E^{\prime}$
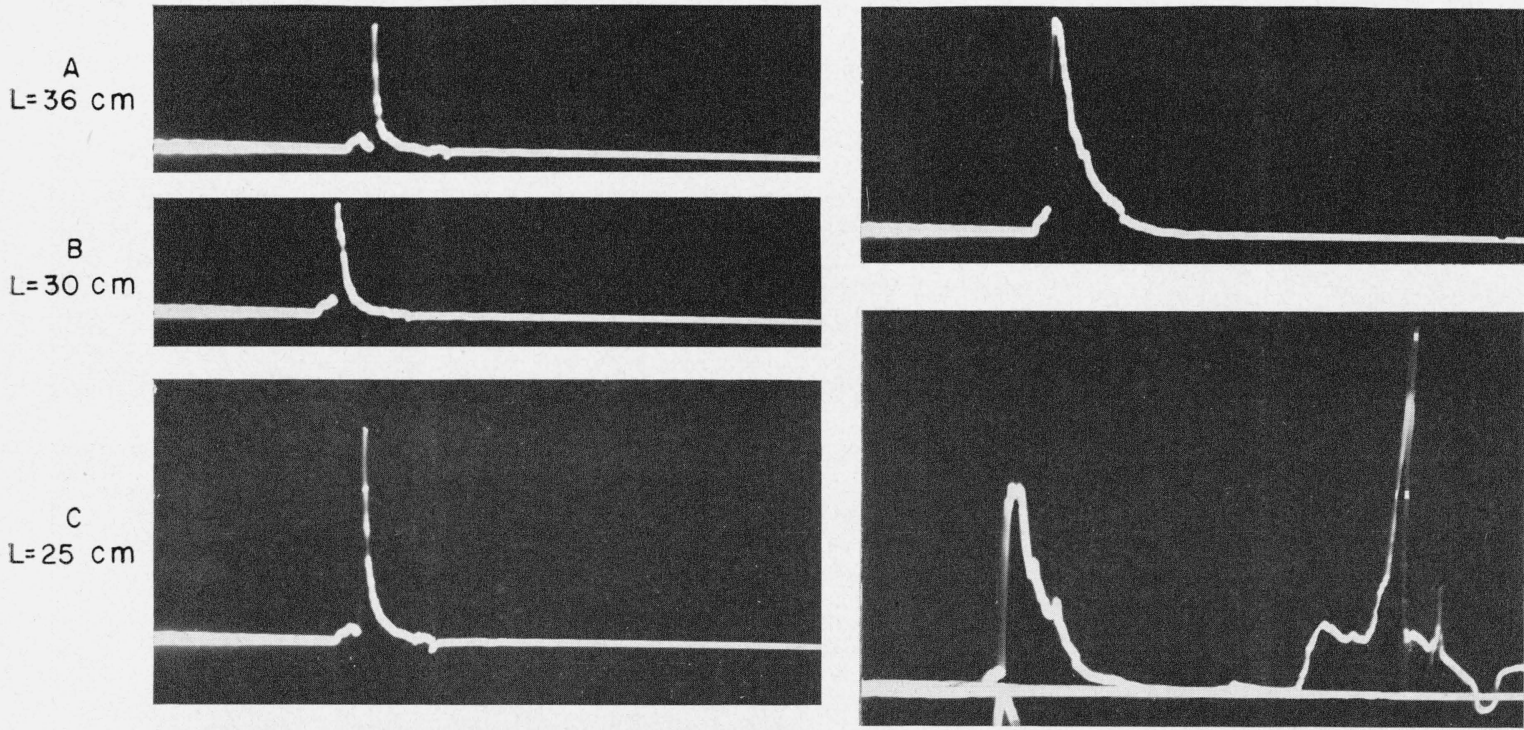

E

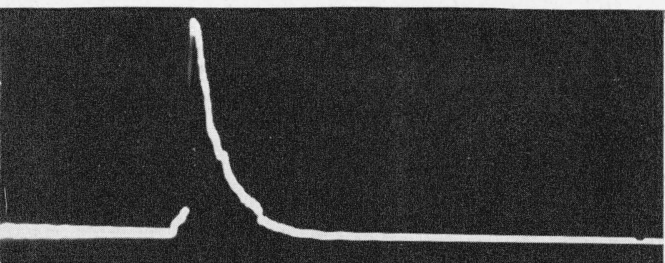

$L=15 \mathrm{~cm}$
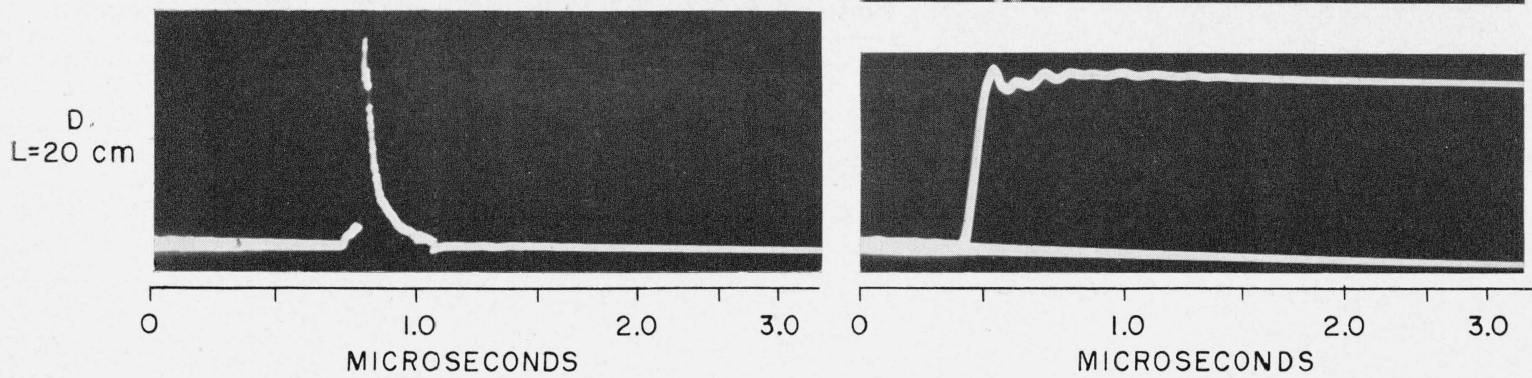

$F$

$L=13 \mathrm{~cm}$

FIGURE 6. Oscillograms of prebreakdown current and discharge pictures taken on the same shots.

Sphere negative, $0.07 \times 100,145-\mathrm{kv}$ surge. Gap spacings $L$ as noted. $\mathrm{F}$ is current oscillogram for complete breakdown of gap. G is oscillogram of applied voltage. 


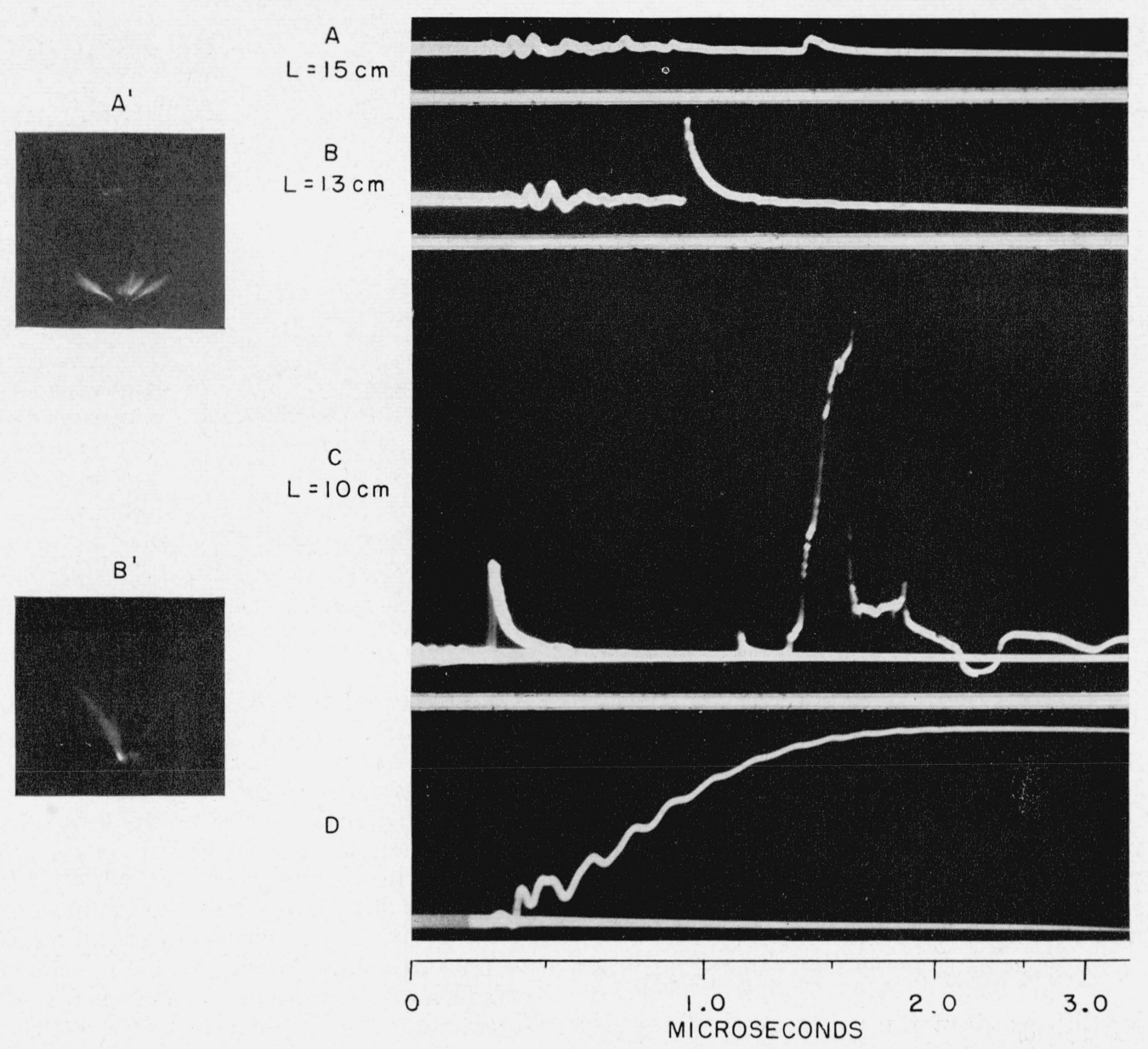

FIGURE 7. Oscillograms of prebreakdown current and discharge pictures taken on the same shots.

Sphere negative, $1 \times 100,145-k v$ surge. Gap spacings $L$ as noted. C is current oscillogram for complete breakdown of gap. D is oscillogram of applied voltage.

"second discharge rise." When the second dis charge rise reaches a value of about $40 \mathrm{amp}$, a safety gap between the sphere and its grounded mounting tube flashes over, thus cutting off the voltage applied to the CRO cable. The corresponding discharge photographs are not included because they show only a large dense streak on the film. Every time the second discharge rise starts, complete breakdown follows unless the voltage wave is chopped. Thus the discharge photographs shown in figures $4,5,6$, and 7 are all associated with the first discharge pip only. It should be noted that although the "streamers" shown in these pictures completely bridge ${ }^{3}$ the gap for spacings of $25 \mathrm{~cm}$ (sphere positive) and $15 \mathrm{~cm}$ (sphere negative) they do not constitute complete breakdown, because after the first discharge pip, the current becomes too low to be measured although the voltage remains high for a much longer time.

3 Complete bridging of the gap was demonstrated by superposing a negative showing the discharge streamers over a negative taken under the same conditions of gap spacing and camera positioning with lights on. Streamers that bridge the gap have a bright spot at their upper tip end as illustrated in figures $4 \mathrm{E}^{\prime}$ and $6 \mathrm{E}^{\prime}$
A large amount of data similar to those illustrated in figures 4 to 7 was accumulated to determine the repeatability of results. The following quantities were tabulated: (1) time delay from start of voltage surge to first discharge pip, (2) peak current value of first discharge pip, (3) percentage of flashovers or breakdowns for a given gap spacing, and (4) time delay from first discharge pip to breakdown. All of these quantities were found to vary from shot to shot and/or to some extent from day to day, some more than others. The various factors that might affect repeatability of results will be considered.

Once the circuit constants were properly adjusted, both the steeply and slowly rising voltage surges repeated very well in waveform from shot to shot and day to day. By careful control of charging voltage, peak values were repeated on successive shots (the measured variations being within 2 percent). Air density and humidity could not be controlled but records were kept of pressure, temperature, and humidity. No attempt was made to determine or apply a correction for air density, but 
for all data used, the relative air density was within 2 percent of that for standard laboratory conditions of $760 \mathrm{~mm}$ of $\mathrm{Hg}$ pressure and $25^{\circ} \mathrm{C}$ temperature. The relative humidity varied from 20 to 75 percent during the period data were being accumulated. The nonrepeatability of experimental results could not be correlated with or accounted for by the small variations in peak magnitude of the applied voltage from shot to shot or by the small changes in relative air density from day to day. There was an indication that changes in relative humidity affected some of the measured quantities.

The surface condition of the spherical electrode and the kind of metal of which the sphere was made may have affected repeatability of results. Most of the data were obtained using a $1.6-\mathrm{cm}$ diameter silver sphere, but data were also obtained using zinc, copper, brass, aluminum, and magnesium spheres of the same diameter. Originally all spheres were given a smooth polish and wiped with a dry chamois, but after even one flashover the surface became quite discolored and after a large number of flashovers the smooth surface was somewhat roughened. Experimental results did not appear to be affected by the condition of the surface of the sphere, but some of the measured quantities appeared to vary slightly with the kind of metal used for the sphere.

Another factor that would be expected to affect repeatability of results is the particular distribution of ions and electrons in the air between the electrodes at the instant voltage is applied. This factor should be of special importance when measuring the time delay from application of voltage to first discharge pip. The conditions prevailing during the experimental observations were intended to approximate average laboratory conditions with each shot being unaffected by previous shots. However, as the gap was located inside a light-tight enclosure so that good pictures of the discharge could be taken, precautions were necessary to insure that the ion distribution was representative of that in free air.

This enclosure had sliding doors at each end. It was found that on the first application of voltage after closing these doors, a discharge pip almost always appeared before peak voltage was reached. It was observed that on succeeding applications of voltage with the doors left closed, either no discharge pip occurred or it was delayed. This can be explained if, during the first application of voltage, a large percentage of the ions inside the enclosure are swept out of the air; because, with the doors closed, there is little chance for new ions to leak into the enclosure before the next application of voltage 30 sec later. When the doors were opened between shots or when a weak gamma-ray source of ionization $\left(\mathrm{Co}^{60}\right.$ giving 0.05 millireontgen $(\mathrm{mr})$ per hour at a distance of $1 \mathrm{~m}$ ) was placed inside the enclosure a discharge pip was always obtained. In order to check the effect of sweeping ions out of the air inside the enclosure, for one series of tests a d-c source of $7,000 \mathrm{v}$ was applied to the upper plane except for the duration of the high-voltage pulse. This sweep voltage effectively prevented a discharge pip with
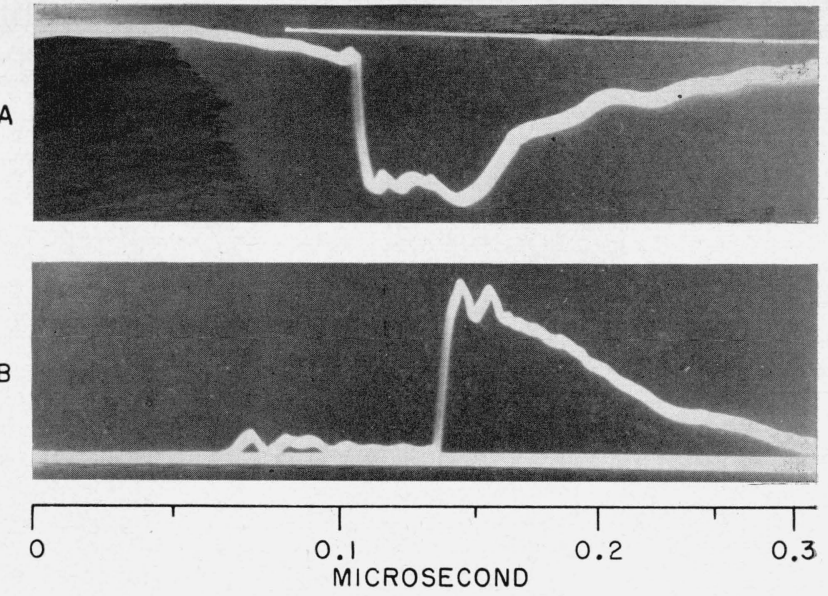

Figure 8. Oscillograms of first discharge current pip on fast sweep.

A, sphere positive $L=23 \mathrm{~cm}$. B, sphere negative $L=15 \mathrm{~cm}$.

the doors closed and the weak ionizing source inside the enclosure. With the doors open the sweep voltage did not prevent the discharge pip, apparently because ions could diffuse into the space between the electrodes faster than they were removed. Most of the experimental results were obtained with the weak $\mathrm{Co}^{60}$ source inside the enclosure, which it was thought would keep ion density inside about equal to that prevailing in the laboratory provided the $7,000 \mathrm{~V}$ was not applied. During some of the experiments stronger ionizing sources were used to study the effect of increasing the ion density in the gap.

A summary of the various quantities that could be tabulated after analyzing the data from over 1,000 shots will now be given. As a start, characteristics associated with the first discharge pip will be described. This discharge appears at gap spacings up to $56 \mathrm{~cm}$ (sphere positive) and $46 \mathrm{~cm}$ (sphere negative), but at the large spacings there is no discharge pip on some of the shots and the time delay values as measured from the initial application of voltage are quite erratic (up to $20 \mu \mathrm{sec}$ ). At gap spacing less than $46 \mathrm{~cm}$ (sphere positive) and $30 \mathrm{~cm}$ (sphere negative) a discharge occurs for nearly every shot, and although the time delay is erratic on an occasional shot it is almost always quite small-less than $0.1 \mu$ sec for the steeply rising surge and less than $1 \mu$ sec for the slowly rising surge.

The wave shape of the first discharge pip is quite repeatable for the sphere positive and somewhat less repeatable for the sphere negative. Typical CRO records of this waveform taken on a fast sweep are shown in figure 8 . In general, an average wave shape can be described which holds for both polarities of the sphere and for both steeply and slowly rising surges. It rises to peak value in about 0.008 $\mu$ sec and decays approximately exponentially to half value in about $0.08 \mu \mathrm{sec}$. This pip-wave shape stays essentially the same for all values of gap spacing but its peak value increases as gap spacing is decreased. 


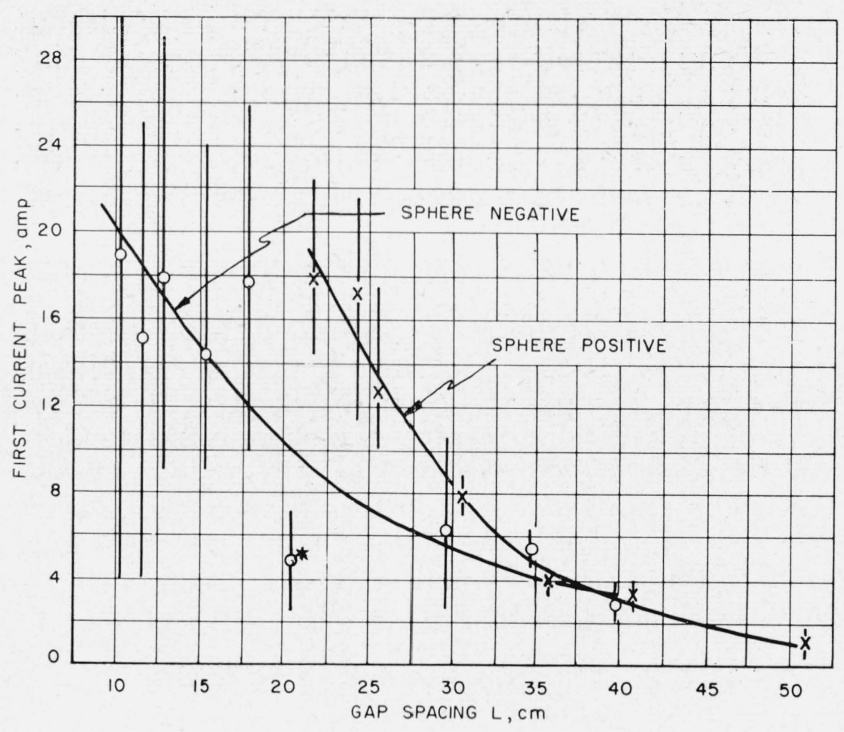

Figure 9. Curves of peak value of first current pip plotted against gap spacing.

$0.07 \times 100,145$-kv surge. Plotted points are each the average of 10 to 100 shots and the vertical lines indicate the spread from minimum to maximum values. *This point is low because on all 10 shots the relative humidity was 75 percent or higher.

The curves in figure 9 are drawn through points of average value of peak current at various gap spacings for the steeply rising surge. Each plotted point is the average of 10 to 100 shots and the spread from the average is indicated by the vertical line through each point. For the sphere positive the points fall quite nicely on a smooth curve, but the spread in values for each point is fairly large - no effect was detected due to changes in relative humidity or the kind of metal used for the sphere. For the sphere negative the spread in values for each point is extremely large, making it difficult to draw definite conclusions; but with the relative humidity above 70 percent, better repeatability and lower values of average current were obtained. Also it was found that zinc or brass spheres gave somewhat higher currents than silver, copper, or magnesium spheres.

Results using the slowly rising surge are not plotted in figure 9. The actual values varied from 1 to 8 amp and could not be correlated with gap spacing. The lower values obtained with slowly rising surges can be accounted for by the fact that the first discharge pip usually starts on the rising front of the surge, frequently at less than half peak value. An analysis of a large number of records obtained with slowly rising surges indicated that the peak current was approximately proportional to the actual value of voltage at the instant the discharge started. Even for the steeply rising surges it is possible that on some shots the discharge started on the rising front of the voltage, thus partially accounting for the spread in values obtained on successive shots. Examination of a large number of pictures of the discharge, such as those shown in figures $4,5,6$, and 7 , indicates a different pattern of tree-like streamers on each shot but in general the

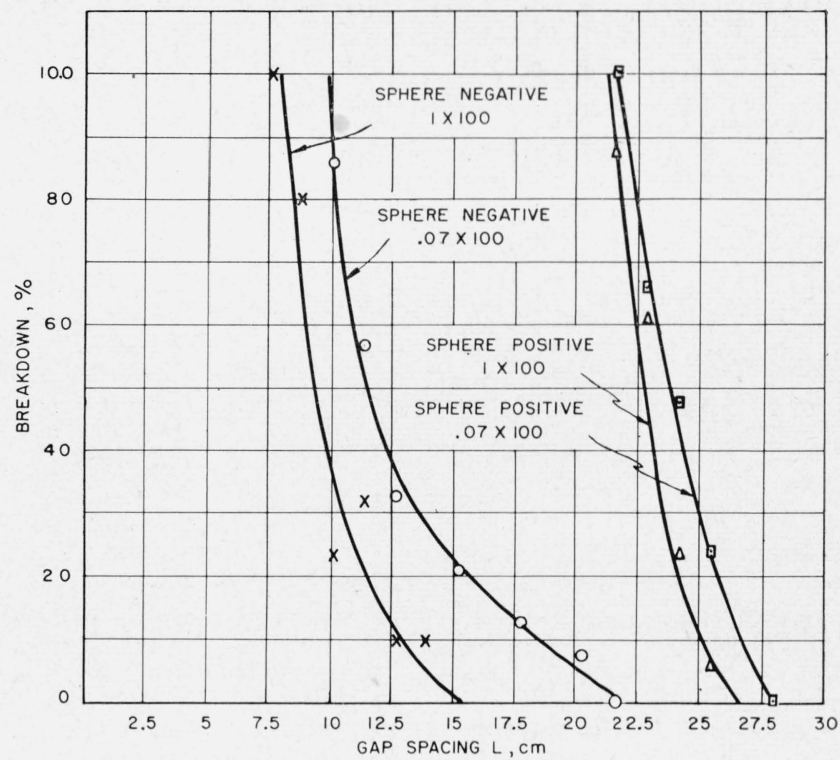

Figure 10. Curves of number of breakdowns expressed as a percentage of the total number of shots at each gap spacing plotted against gap spacing.

length of these streamers increases in proportion to the peak discharge current. These prebreakdown streamers completely bridge the gap for spacings at which breakdown is likely.

For gap spacings less than $28 \mathrm{~cm}$ (sphere positive) or $20 \mathrm{~cm}$ (sphere negative), complete breakdown occurs for at least some shots and there is a time delay from the first discharge pip to breakdown as indicated in figures $4 \mathrm{~F}, 5 \mathrm{E}, 6 \mathrm{~F}$, and $7 \mathrm{C}$. A large number of shots at gap spacings giving breakdown were taken using silver, zinc, and copper spheres. The kind of metal used for the sphere and its surface condition may have had an effect, but because of the statistical nature of the results this effect could not be definitely established and the data on silver, zinc, and copper spheres were all averaged together.

The curves in figure 10 show the number of breakdowns as a percentage of the total number of shots at each gap spacing plotted against gap spacing. Each plotted point represents the results of at least 50 shots. For the sphere positive the increase in gap spacing in going from 100 to 0 percent flashover is 30 percent for the steeply rising surge and 25 percent for the slowly rising surge. For the sphere negative this increase is 120 percent for the steeply rising surge and 90 percent for the slowly rising surge. Also, for sphere positive the gap spacing giving 100 percent flashover is very nearly the same for both the steeply and slowly rising surges, but for sphere negative the gap spacing is $9.6 \mathrm{~cm}$ for the steeply rising surge and $8.1 \mathrm{~cm}$ for the slowly rising surge. Thus breakdown occurs over a wider range of gap setting for the sphere negative than for the sphere positive and a steeply rising surge increases this range for both polarities.

A plot of time delay from start of first discharge pip to breakdown, against gap spacing is given in 


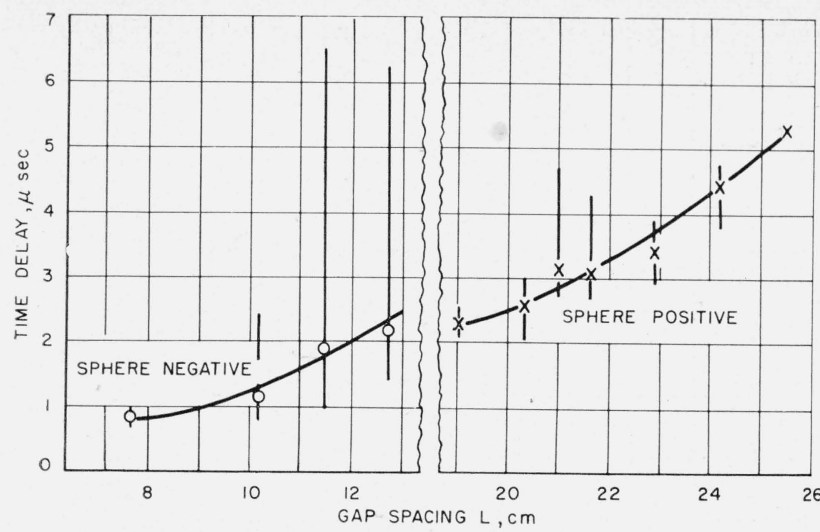

Figure 11. Curves of time delay from start of first current pip to breakdown plotted against gap spacing.

$0.07 \times 10$ 9, $145-\mathrm{kv}$ surge. Plotted points are each the average of 10 to 30 shots and the vertical lines indicate the spread from minimum to maximum values.

figure 11 for a steeply rising surge. Each of the plotted points through which the curves are drawn is the average of 10 to 30 shots and the vertical line through each point shows the spread from minimum to maximum time delay at that spacing. The time delay increases on the average with gap spacing but the variation from shot to shot at each gap spacing is quite large, especially with the sphere negative. Time delays for the slowly rising surge are not plotted, but the average values follow about the same curves as plotted in figure 11 and the variations from shot to shot at each gap spacing are about the same.

The statistical nature of the experimental results might be caused by variations in the distribution of free electrons and ions in the gap from shot to shot. As already mentioned, a weak ionizing source was used while obtaining the above data in order to give an average ion density inside the gap enclosure that would correspond to average laboratory conditions outside of such an enclosure. However, the ion distribution still varied from instant to instant and it would be very difficult, if not impossible, to control the instantaneous distribution.

The distribution can be greatly modified by using a strong artificial source of ionization. This was done in two ways: (1) by placing a strong gamma-ray source $\left(\mathrm{Co}^{60}\right.$ giving $50 \mathrm{mr} / \mathrm{hr}$ at $1 \mathrm{~m}$ ) about $46 \mathrm{~cm}$ below the sphere with its beam aimed at the sphere, or (2) by placing a weaker source $\left(\mathrm{Co}^{60}\right.$ giving 0.5 $\mathrm{mr} / \mathrm{hr}$ at $1 \mathrm{~m}$ ) inside the sphere and near its upper surface.

Data similar to those described above were taken with either one or the other of these gamma-ray sources in place. The following results were noted: (1) the first discharge pip appeared on every shot, even at gap spacings up to $56 \mathrm{~cm}$ (sphere positive), and its time delay even at the long gap spacings was always less than $0.2 \mu \mathrm{sec}$; (2) the value of peak current of the first discharge pip was much more repeatable from shot to shot, the gain in repeatability being very pronounced for a steeply rising surge and sphere negative; (3) the average value of peak current was less - down to about one-third its value without the strong source for sphere negative at gap spacing $10 \mathrm{~cm}$; (4) the gamma-ray source had little or no effect on the time lag of breakdown versus gapspacing curves; (5) the only noticeable effect on the percent breakdown versus gap-spacing curves was that for the slowly rising surge and sphere negative no breakdown occurred at gap spacings greater than $11.4 \mathrm{~cm}$.

From these results it is concluded that variations from shot to shot, (1) in the time delay to start of first discharge pip and (2) in the peak value of current of first discharge pip, arise from the random distributions of charges in the gap at the instant voltage is applied. Once the first discharge pip has occurred, however, the subsequent mechanism of breakdown is not affected to any great extent by this initial charge distribution.

\subsection{Chopped-Wave 145-kv Surge}

Further investigation of the discharge that occurs during the first discharge pip was carried out by chopping the voltage very quickly after the first discharge pip starts. A large number of oscillograms of the current showing the first discharge pip, and corresponding pictures of the discharge, were taken with chopping time from 0.005 to $0.02 \mu$ sec after start of first discharge pip. Typical examples of these records are shown in figure 12 for sphere positive and figure 13 for sphere negative. Some branches of the streamers propagate across the gap very quickly (in about $0.01 \mu \mathrm{sec}$ ), and their appearance is very nearly the same as those obtained without chopping the voltage wave but at greater gap spacings. An approximate value for the velocity of propagation of these streamers was obtained by determining the actual length of the streamers from a discharge picture, and taking the time of formation from the corresponding current oscillogram as the time from start of discharge pip to time voltage was chopped. This was done for 10 sets of records for each polarity (similar to those shown in figs. 12 and 13). The mean streamer propagation velocity was found to be $500 \mathrm{~cm} / \mu \mathrm{sec}$ for sphere negative and 800 $\mathrm{cm} / \mu$ sec for sphere positive, the average deviations being 90 for sphere negative and 100 for sphere positive.

The experimental results obtained using a full-wave surge voltage indicate that the discharge is initiated by a sudden burst of current, which is quickly reduced to a low value and remains near zero unless complete breakdown is to occur. When and only when breakdown is to occur, the discharge current begins to increase again (the "second discharge rise"), rather slowly at first and then at an ever increasing rate up to breakdown. If breakdown is complete, a picture of the discharge is of little value because the film is greatly overexposed by the intense light from the high current arc. If, however, the voltage wave is chopped off at some time before complete breakdown would have occurred, a photograph of the discharge should be indicative of much of the ionization phenomena taking place up to the time of chopping. 

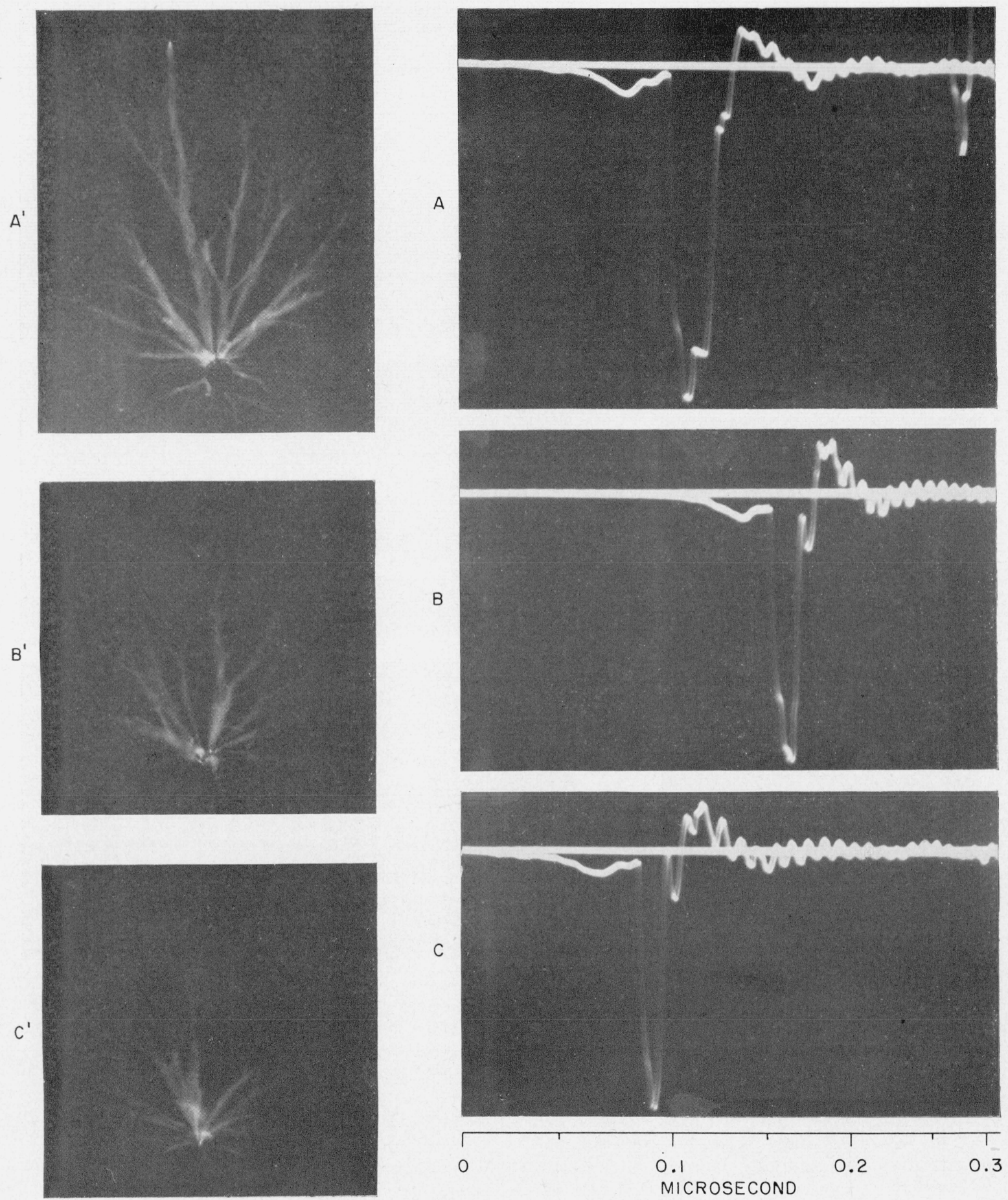

Figure 12. Oscillograms of first current pip $(A, B$, and $C)$ and discharge pictures $\left(A^{\prime}, B^{\prime}\right.$, and $\left.C^{\prime}\right)$ on the same shots with voltage wave chopped quickly after start of first current pip.

$0.07 \times t_{c}, 145-\mathrm{kv}$ surge, sphere positive. Gap spacing $=18 \mathrm{~cm}$ for $\mathrm{A}$ and $\mathrm{B}$ and $20 \mathrm{~cm}$ for $\mathrm{C}$. 

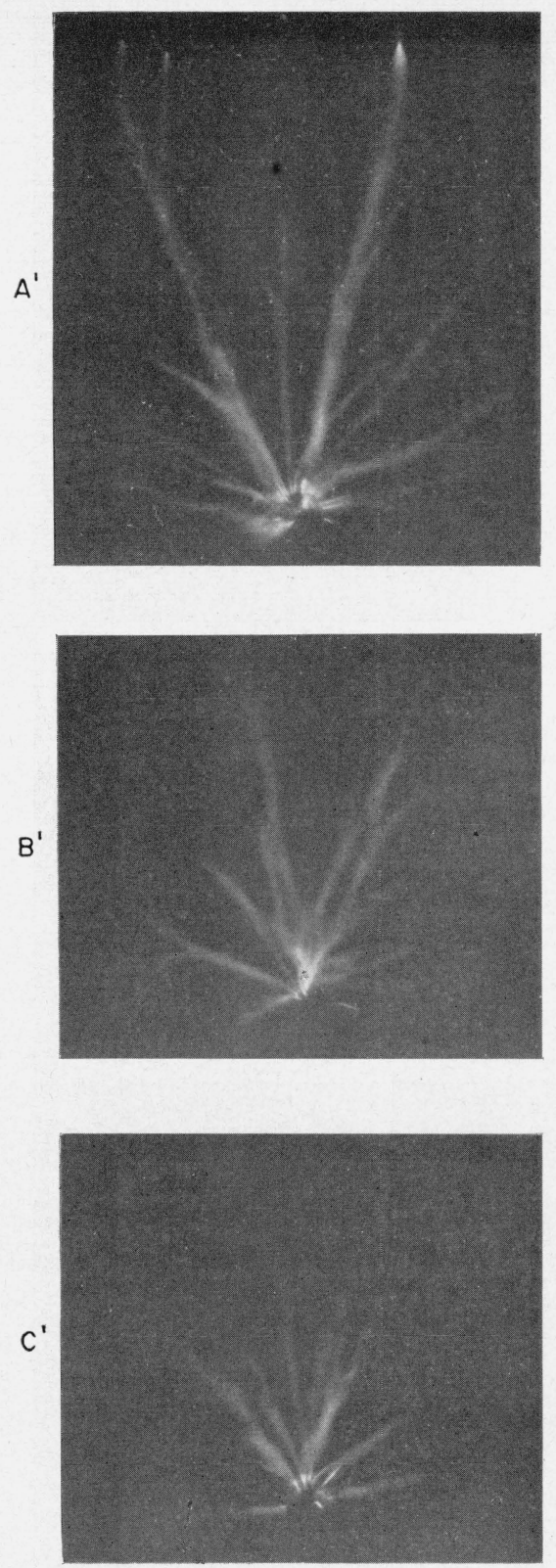

Figure 13. Oscillograms of first current pip $(A, B$, and $C)$ and discharge pictures $\left(A^{\prime}\right.$, $B^{\prime}$, and $\left.C^{\prime}\right)$ on the same shots with voltage wave chopped quickly after start of first current pip.

$0.07 \times t_{c} .145$-surge, sphere negative. Gap spacing $L=15 \mathrm{~cm}$.

Such records were obtained by using the chopping circuit described in section 3 . Oscillograms of discharge current and pictures of the discharge on the corresponding shot were taken at various chopping times. For these tests, the gap spacing $L$ was held constant at a value that would nearly always give complete breakdown for a full-wave applied surge. Results were obtained for both polarities of the sphere and for both steeply and slowly rising surges. Because sphere polarity had a marked effect, a description of results for each polarity will be given separately.

The oscillograms of discharge currents (figs. 14A to $14 \mathrm{~F}$ ) and the corresponding pictures of the discharge (figs. $14 \mathrm{~A}^{\prime}$ to $14 \mathrm{~F}^{\prime}$ ) were obtained using $0.07 \times t_{c}, 145$-kv surges with the sphere positive and a gap spacing of $20 \mathrm{~cm}$. Each picture is, of course, of a separate shot, so that the streamer pattern should not be expected to repeat in exact detail from picture to picture. Rather, this series of pictures has been 
$A^{\prime}$

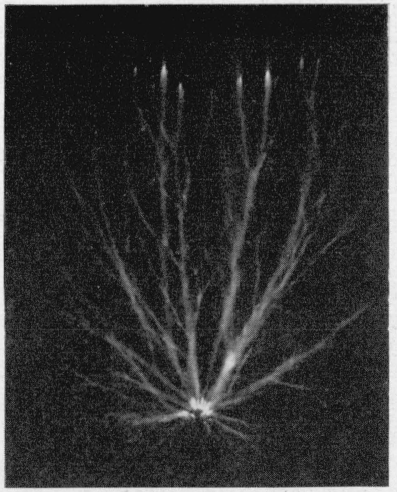

$D^{\prime}$

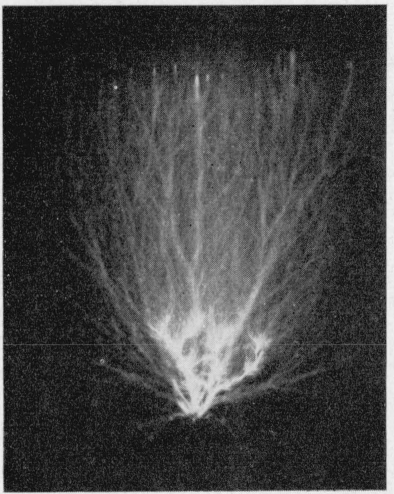

A

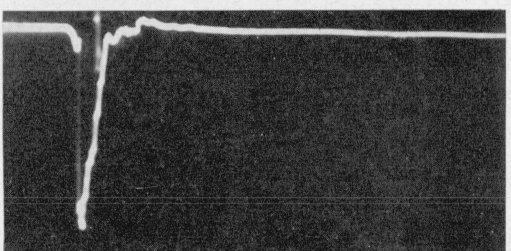

B

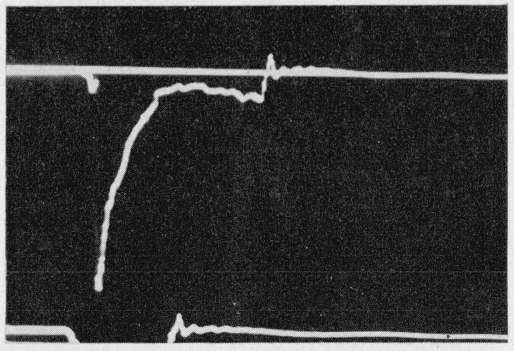

C
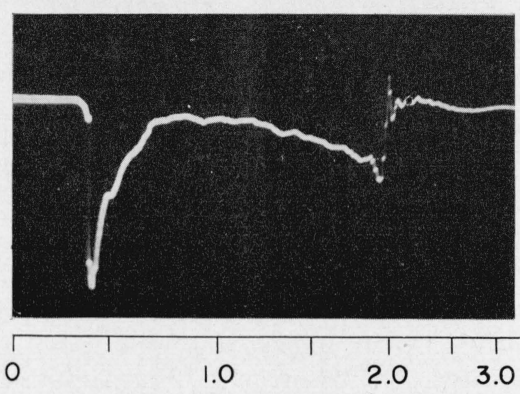

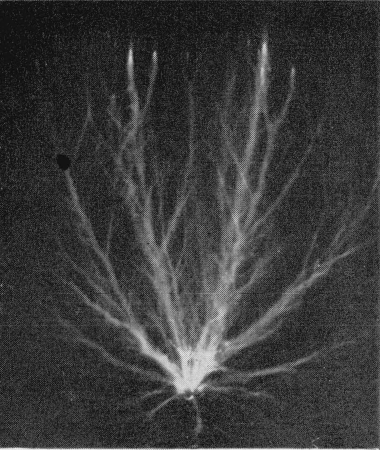

$B^{\prime}$

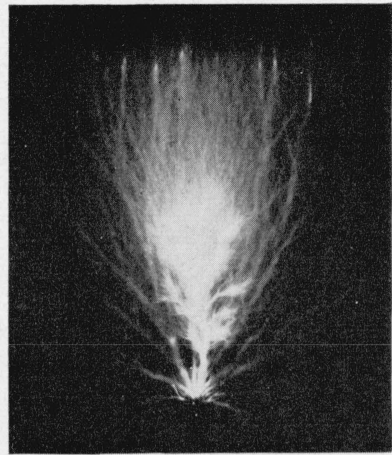

$E^{\prime}$
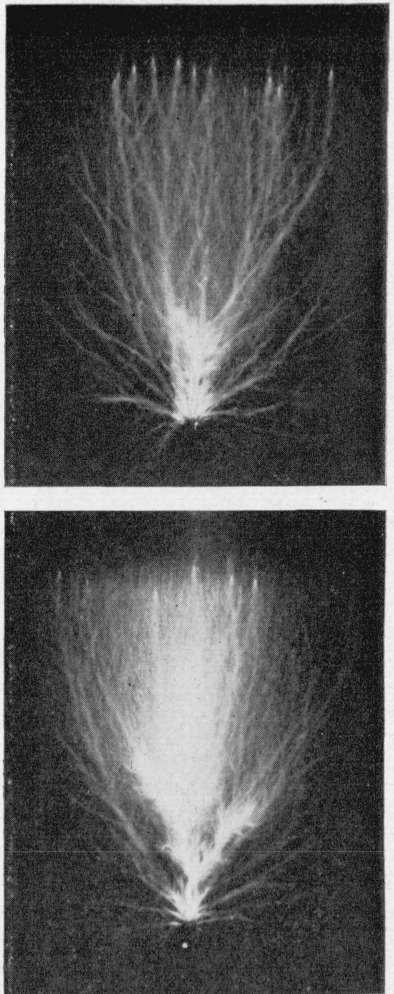

F

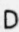

E
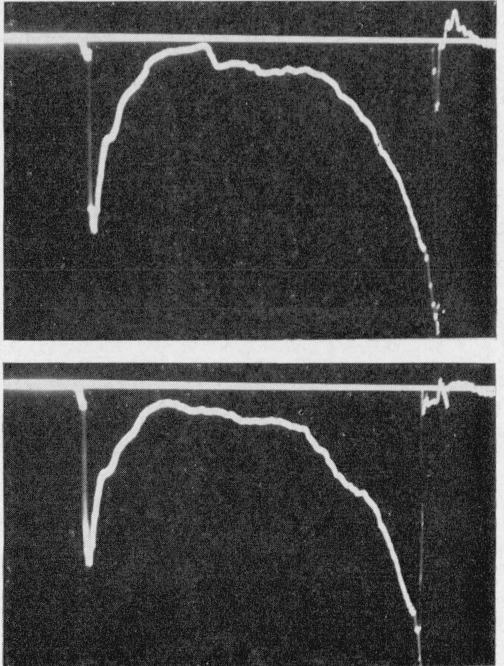

F

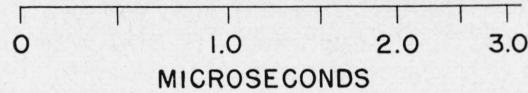

FIGURE 14. Oscillograms of prebreakdown current and discharge pictures taken on the same shots with applied voltage chopped at various times after the first current pip.

$0.07 \times t_{c}, 145-\mathrm{kv}$ surge (see figure $4 \mathrm{G}$ ), sphere positive. Gap spacing $20 \mathrm{~cm}$-breakdown would be complete if voltage were not chopped. 


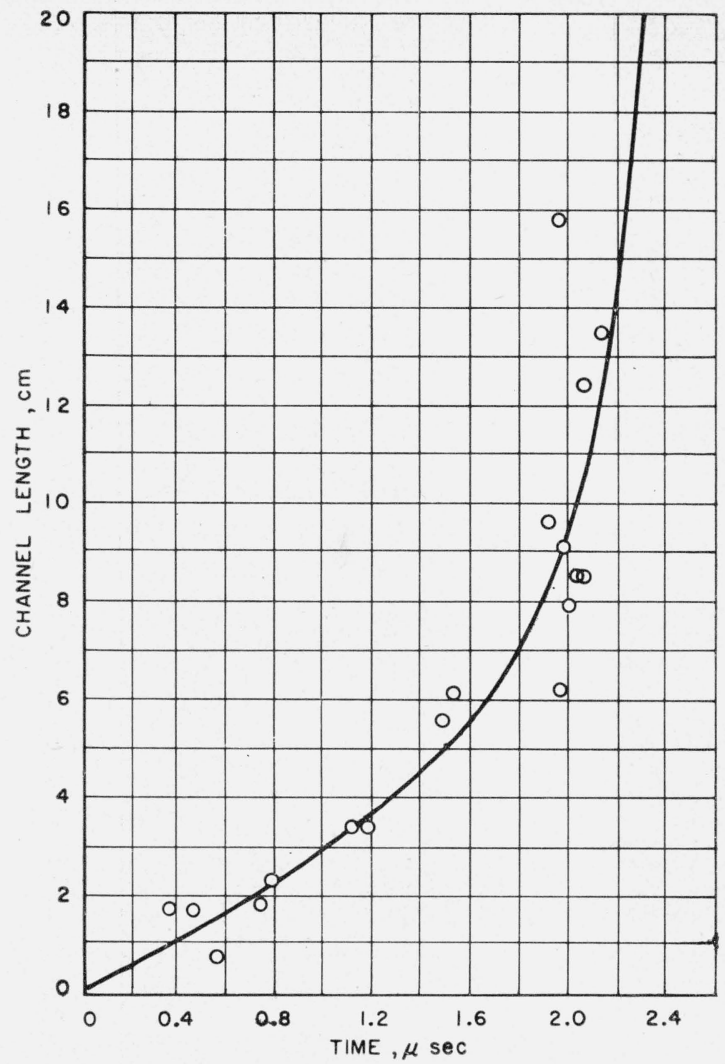

Figure 15. Plot of channel length against time from first current pip to chopping.

$0.07 \times t_{c}, 145-\mathrm{kv}$ surge. Sphere positive. $L=20 \mathrm{~cm}$.

chosen to show the discharge as nearly as possible as it would appear at successive intervals after the first current pip. The initial streamer patterns that form during the first discharge pip and before the second discharge rise starts (see figs. $14 \mathrm{~A}$ and $14 \mathrm{~A}^{\prime}$ ) are very similar in appearance to those previously obtained when the voltage was not chopped and breakdown did not occur (see figs. $4 \mathrm{E}$ and $4 \mathrm{E}^{\prime}$ ). The appearance of the discharge begins to change after the second discharge rise starts. This change consists of one or more bright discharge channels starting at or near the sphere. As the discharge current increases, these brilliant channels appear to extend in a rather tortuous path toward the plane electrode. Each bright tufted channel appears to be fed by a multitude of very fine hair-like filaments. These hair-like filaments are continuously developed from the leading fingers or branches of the growing channel and many appear to extend all the way to the plane electrode very early in the channel growth.

Approximate values for the rate of channel growth were obtained by measuring (1) the channel length on a discharge picture and (2) the time from first discharge pip to the chopping of the voltage wave on the corresponding current oscillogram. This was done for 18 sets of records similar to those shown in figure 14. A plot was then made of channel length against time to chopping of the voltage wave, and a smooth curve drawn through the plotted points as shown in figure 15. The slope of this curve gives approximate values for the instantaneous rate of channel growth. The channel starts to grow at a fairly slow and uniform rate of $3 \mathrm{~cm} / \mu \mathrm{sec}$. After it has reached a length of $3 \mathrm{~cm}$ it grows at an increasing rate. At midgap $(10 \mathrm{~cm})$ its rate of growth is about $20 \mathrm{~cm} / \mu \mathrm{sec}$. Beyond midgap it appears to grow much faster, and quickly reaches the plane electrode, causing complete breakdown.

Current oscillograms and discharge pictures shown in figure 16 were obtained in the same manner as those in figure 14, except that the slowly rising surge voltage $\left(1 \times t_{c}, 145-\mathrm{kv}\right)$ was used. Breakdown occurs in essentially the same way, but the initial streamers that develop during the first current pip vary greatly in pattern and magnitude from shot to shot. As seen from figures $16 \mathrm{~A}, 16 \mathrm{~B}$, and $16 \mathrm{D}$, when the first discharge pip appears after the applied voltage has risen to a fairly large value, its magnitude is quite large and the initial streamers are fully developed and similar to those in figure 14 . As seen from figures $16 \mathrm{C}, 16 \mathrm{E}$, and $16 \mathrm{~F}$, when the first discharge pip appears before the applied voltage has reached a high value its magnitude is much less and the initial streamers do not extend very far from the sphere. However, the growth of the channel which leads to breakdown does not appear to be greatly affected by the magnitude or pattern of the initial streamers.

With the sphere negative, current oscillograms and discharge pictures for the voltage chopped at various times are shown in figures 17 and 18 . The time delay from first discharge pip to breakdown varies more from shot to shot than with the sphere positive, and this increased the experimental difficulty in obtaining a set of illustrative records with the voltage chopped at various stages in the breakdown process. However, fairly satisfactory sets of records were obtained by taking a large number of shots. The initial discharge streamers bridge the gap on all shots for a steeply rising voltage (fig. 17), and on some shots for the slower rising voltage (fig. 18). Breakdown begins with the second discharge rise, and the increase in this current is not as gradual as for sphere positive. At about the same time as the second discharge rise starts, the discharge pictures show positive channels (plasma) beginning from the plane. These were noted by Norinder [10, fig. 16, p. 510] and referred to as "plate stems." These plane or anode channels grow toward the sphere in much the same way that a channel starting from the sphere grows toward the plane with sphere positive. As the channel approaches the sphere, breakdown occurs very rapidly, when the channel either contacts the sphere or connects with a channel developing from the sphere. 

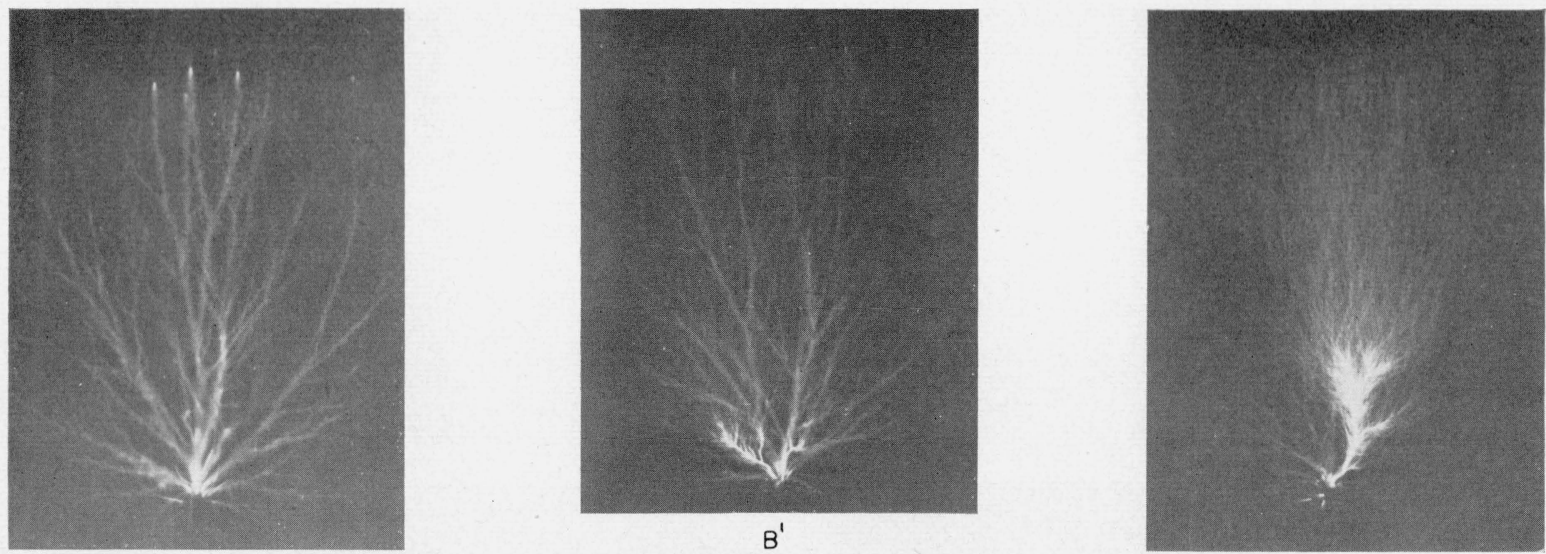

$B^{\prime}$

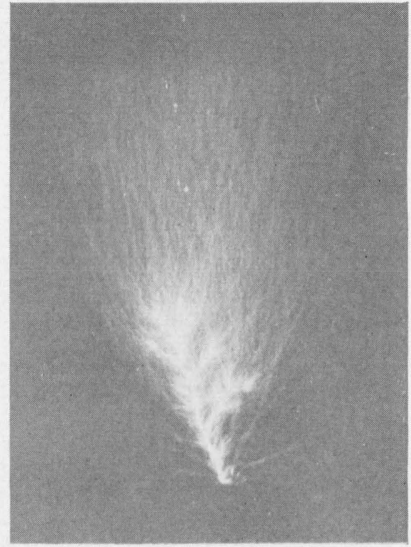

$F^{\prime}$

$D^{\prime}$

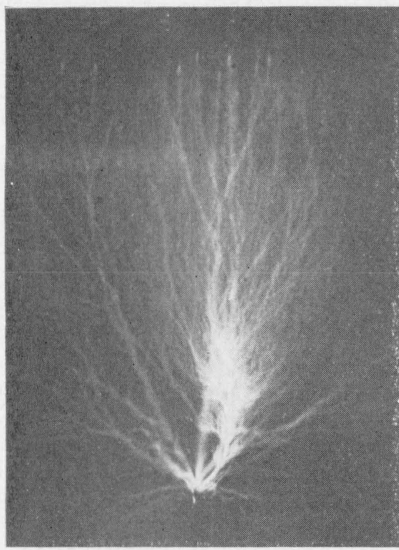

A

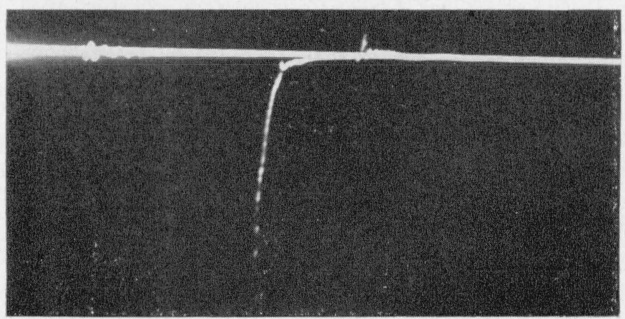

B
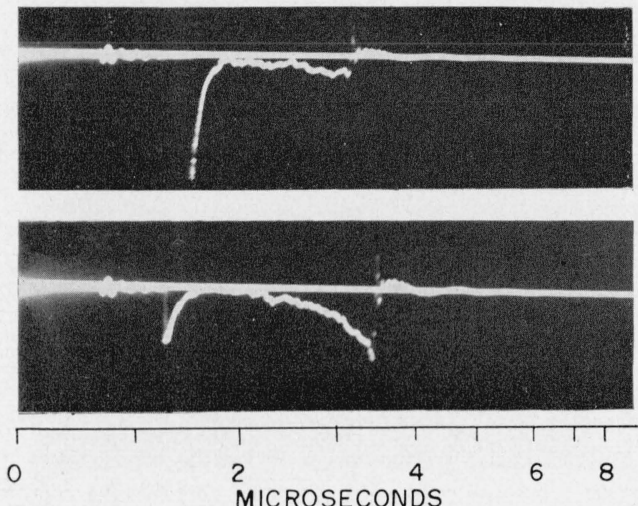

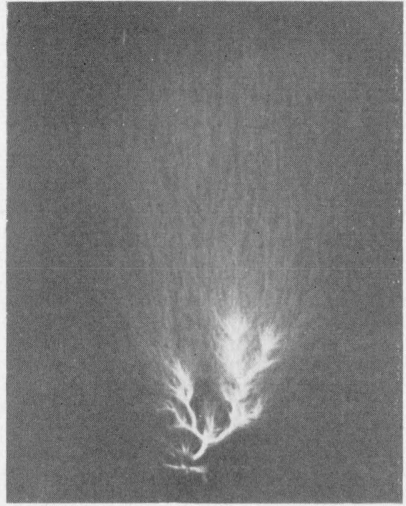

$E^{\prime}$
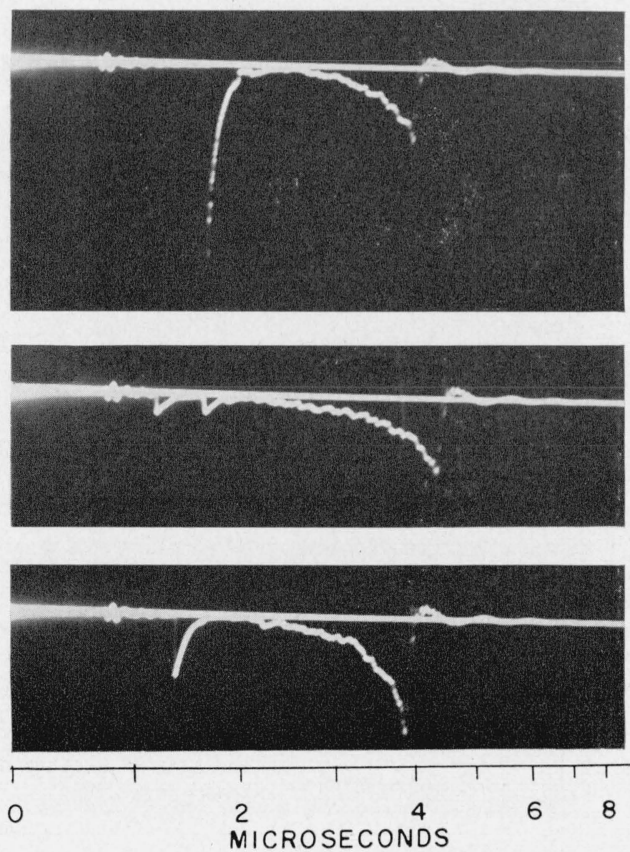

FiguRE 16. Oscillograms of prebreakdown current and discharge pictures taken on the same shots with applied voltage chopped at various times after first current pip.

$1 \times t_{c}, 145 \mathrm{kv}$ surge (see figure $5 \mathrm{~F}$ ), sphere positive. Gap spacing $20 \mathrm{~cm}$-breakdown would be complete if voltage were not chopped. 

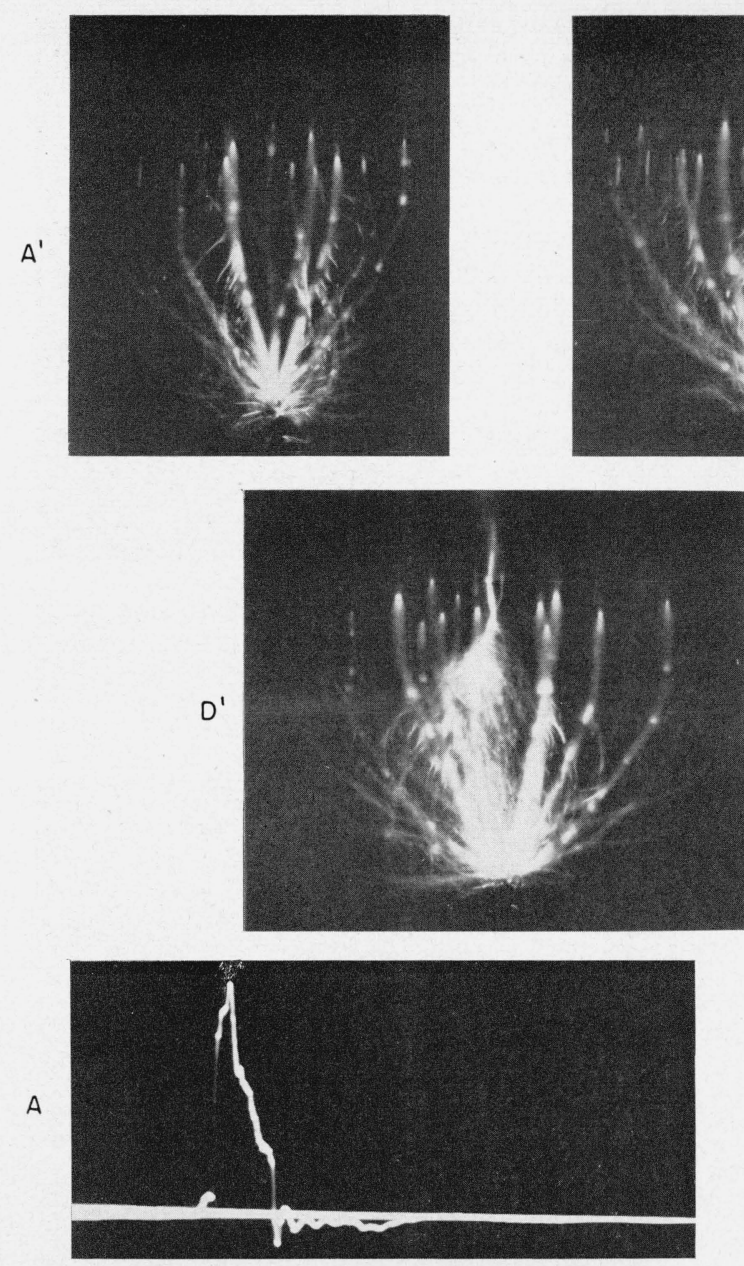

B

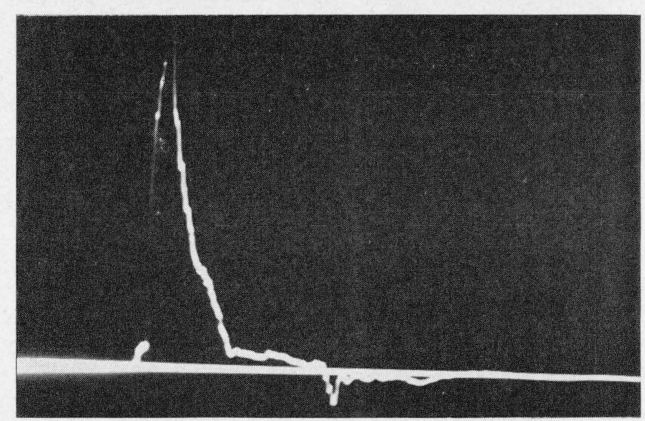

C

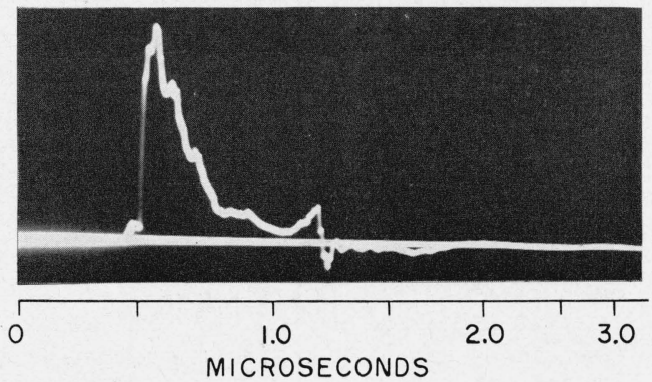

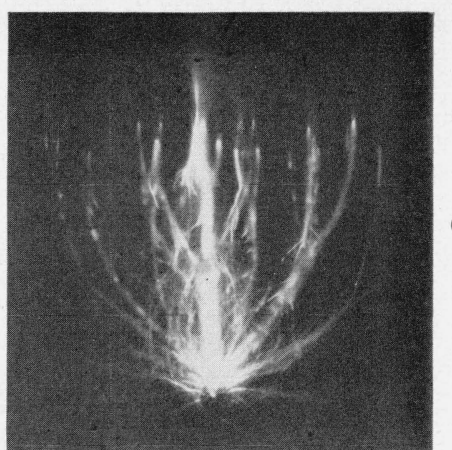

$C^{\prime}$

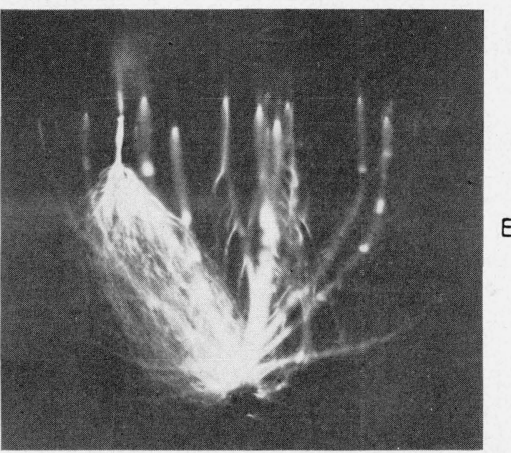

D
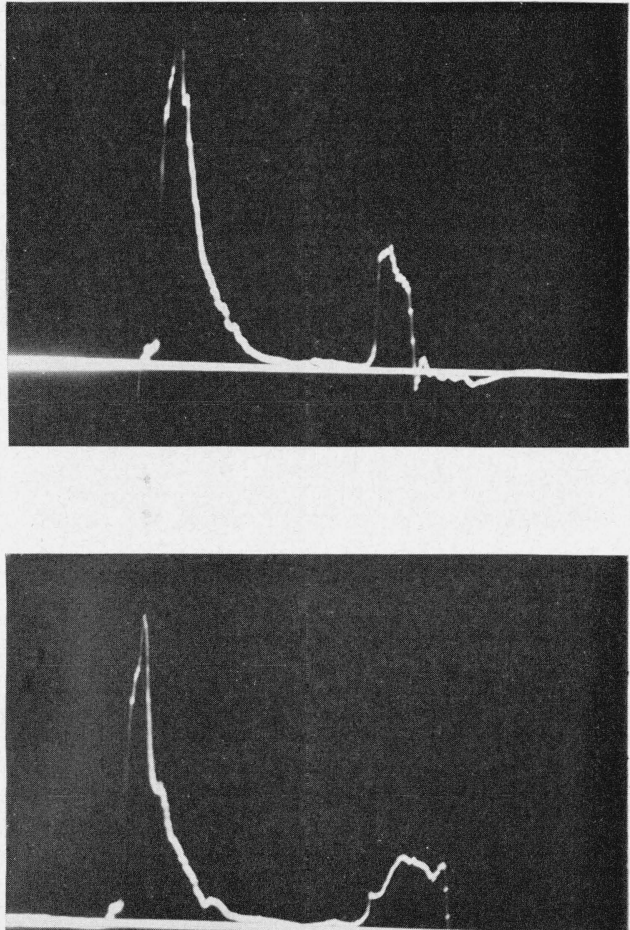

$E$

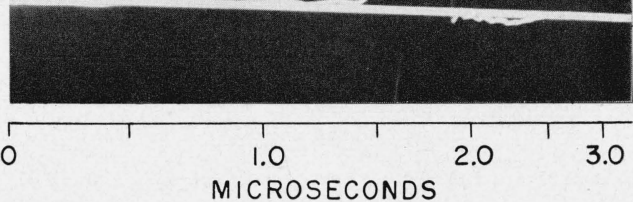

MICROSECONDS

Figure 17. Oscillograms of prebreakdown current and discharge pictures taken on the same shots with applied voltage chopped at various times after first current pip.

$0.07 \times t_{c}, 145-\mathrm{kv}$ surge (see figure $6 \mathrm{G}$ ), sphere negative. Gap spacing $11.5 \mathrm{~cm}$-breakdown would be complete if voltage were not chopped. 

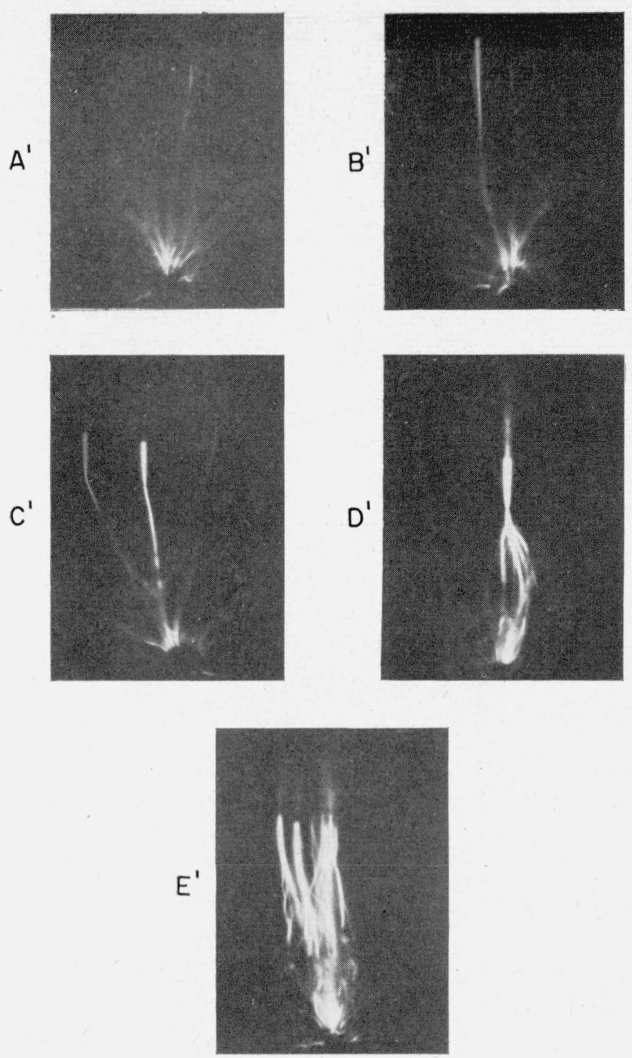

A

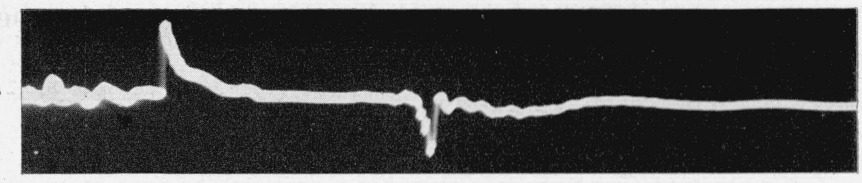

B

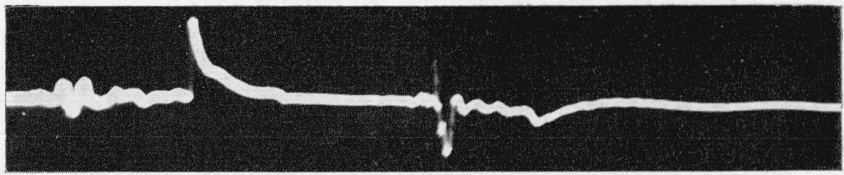

C

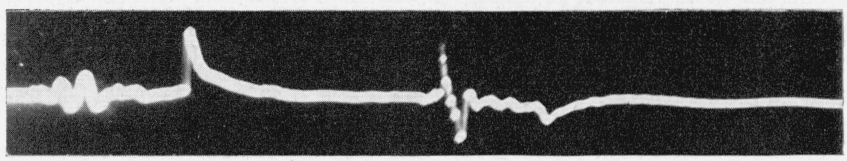

D

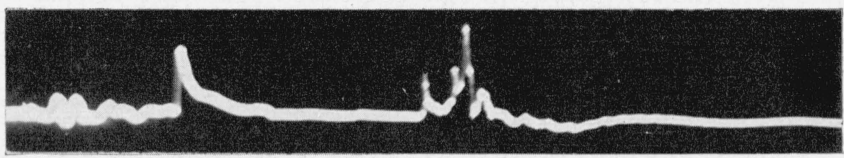

$E$

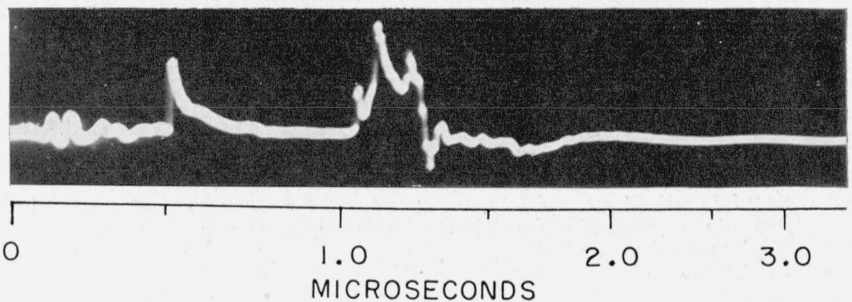

FIGURE 18. Oscillograms of prebreakdown current and discharge pictures taken on the same shots with applied voltage chopped at various times after first current pip.

$1 \times t_{c}, 145-\mathrm{kv}$ surge (see figure 7D), sphere negative. Gap spacing $7.5 \mathrm{~cm}$-breakdown would be complete if voltage wave were not chopped.

\section{Analysis of Experimental Results}

The reader may feel the need of referring to particular discharge pictures and current oscillograms while going over this last section. Table 1 summarizes these records and gives the corresponding figure numbers.

TABLE 1. Summary reference tabulation of discharge picture an current oscillogram figures

\begin{tabular}{|c|c|c|c|}
\hline Sphere polarity & Wave front & Wave tail & $\begin{array}{c}\text { Figure } \\
\text { No. }\end{array}$ \\
\hline $\begin{array}{l}\text { Positive } \\
\text { Do } \\
\text { Do } \\
\text { Do } \\
\text { Do } \\
\text { Negative } \\
\text { Do } \\
\text { Do } \\
\text { Do } \\
\text { Do }\end{array}$ & $\begin{array}{c}\mu s e c \\
0.07 \\
.07 \\
.07 \\
1.0 \\
1.0 \\
.07 \\
.07 \\
.07 \\
1.0 \\
1.0\end{array}$ & $\begin{array}{l}100 \mu \mathrm{sec} \\
\text { Chopped very quickly } \\
\text { Chopped after } 0.5 \mu \mathrm{sec} \\
100 \mu \mathrm{sec} \\
\text { Chopped after } 1 \mu \mathrm{sec} \\
100 \mu \mathrm{sec} \\
\text { Chopped very quickly } \\
\text { Chopped after } 0.5 \mu \mathrm{sec} \\
100 \mu \text { sec } \\
\text { Chopped after } 1 \mu \mathrm{sec}\end{array}$ & $\begin{array}{r}4 \\
12 \\
14 \\
5 \\
16 \\
6 \\
13 \\
17 \\
7 \\
18\end{array}$ \\
\hline
\end{tabular}

\subsection{First Discharge Pip}

In order to explain the initial discharge streamers and the current pip associated with them, it is necessary to make some additions and modifications to theories already postulated. Because of their high velocity of formation and the fact that they follow the lines of force of the applied field, these streamers cannot be compared to the pilot streamer or stepped leader stroke observed in lightning. Because they can travel across the gap without leading to breakdown they cannot be completely explained by Loeb and Meek's streamer theory [5], although this theory will be used here as the basis for their explanation. A brief summary of the proposed explanation will be given first, followed by details of various phases of the mechanism involved and correlation with the experimental data.

The term "streamers" is used because of their appearance on the discharge pictures. Actually these photographic traces (which will still be called streamers) are produced by balls or regions of high space-charge density with high ionization and excitation activity at their leading surface, being projected from the sphere toward the plane along lines of force of the applied field. If moving pictures at a speed of $10^{10}$ frames per second could be taken, each frame would presumably show a small localized bright area somewhere between the sphere and the plane, while regions outside the ball would give very little light. The ball does not consist of a group of 
electrons or ions moving outward from the sphere, ${ }^{4}$ but should be thought of as a traveling wave of high charge density which is propagated by a process in which new charges are continually produced at the leading surface of the ball by the high gradient there. In the path behind the ball there is left a high concentration of both positive and negative ions, with an excess of positive ions in case the sphere is positive and an excess of negative ions in case the sphere is negative. The net space charge left by the moving ball is enough to markedly reduce the original high gradient near the sphere, and thus inhibit for a short time further ionization activity in the path behind the ball. The streamers do not form conducting paths from the sphere to the plane because after the ball has crossed the gap the residual gradients are so low that no more ions are produced.

To explain the formation and propagation of the ball of high charge density, a process is suggested similar to that used by Loeb and Meek [5] in their streamer theory for uniform fields, which starts with the familiar electron avalanche. The chance of a free electron being correctly positioned with respect to the sphere to start an avalanche at the instant at which voltage is applied is quite small. But it has been demonstrated that the streamers do start at or very nearly at this instant for a gap out in the open under laboratory conditions, giving an average background density of ions in the air between the electrodes. Hence a free electron for avalanche initiation is probably produced from a negative ion by the high voltage gradient $(90 \mathrm{kv} / \mathrm{cm}$ or more) near the sphere when voltage is applied. With the sphere positive a ball of positive space charge is formed by an avalanche, and with the sphere negative a ball of negative space charge. The two cases will be considered separately.

With the sphere positive, a negative ion situated in the high-field region near the sphere supplies a free electron, which starts ionizing neutral molecules and quickly forms an avalanche. As soon as this ionization process starts, it furnishes a plentiful supply of photons which liberate electrons in the nearby high field, where they, too, can readily start new avalanches. By means of this speedy relay of photons over the entire high-field region adjacent to the sphere, its upper half becomes rapidly "peppered" with avalanches.

The electrons are drawn out of the avalanches into the sphere, leaving balls of high positive spacecharge density near the surface of the sphere. These balls will, of course, vary in size and charge density, but at least some of them will be capable of initiating positive streamers. ${ }^{5}$ The space-charge balls enhance

\footnotetext{
${ }_{4}$ The experiments show that initial streamers travel the entire gap length in times of about $10^{-8}$ seconds (measured velocities of propagation being from 500 to $1,000 \mathrm{~cm} / \mu \mathrm{sec})$. As the drift velocity of electrons in a region of high gradient $(100 \mathrm{kv} / \mathrm{cm})$ is only about $20 \mathrm{~cm} / \mu \mathrm{sec}$, and that of ions less than $0.2 \mathrm{~cm} / \mu \mathrm{sec}$, the $(100 \mathrm{kV} / \mathrm{cm})$ is only about $20 \mathrm{~cm} / \mu \mathrm{sec}$, and that of ions less than $0.2 \mathrm{~cm} / \mu \mathrm{sec}$, the
process of streamer formation cannot be explained by ions or electrons moving process of streamer form

along its entire length. large number. An estimate of the total number of streamers that bridge the gap on a given shot can be obtained by counting the bright spots at the plane end of the gap on the discharge picture and multiplying by three, the factor three entering because of the depth of focus of the lens. (The lens has a focal length of $58 \mathrm{~mm}$, and the maximum aperture of $\mathrm{f} / 1.9$ was used with the camera at a distance of 76 $\mathrm{cm}$ from the gap, the depth of focus thus being $2.5 \mathrm{~cm}$.) The number of streamers and their patterns vary greatly from shot to shot but in general for a given applied voltage the shorter the gap the more streamers. For the sphere positive, a gap
}

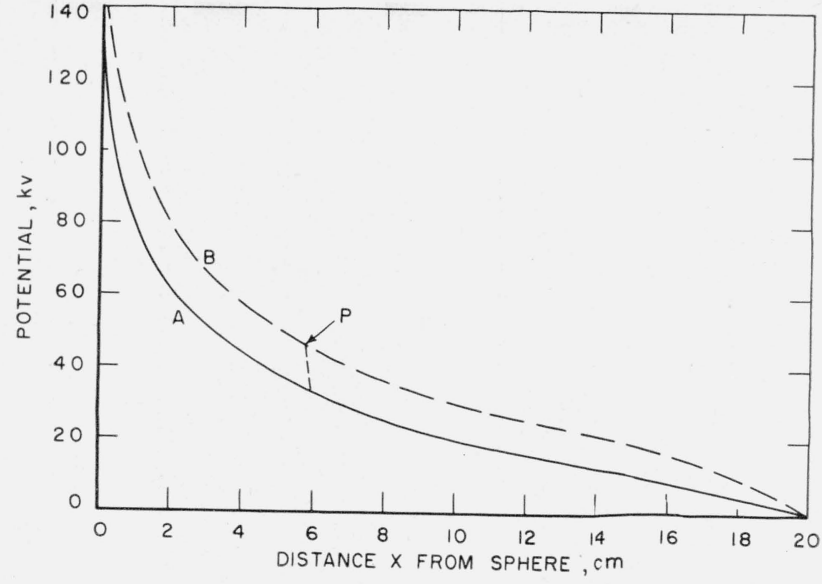

Figure 19. Potential distribution along a streamer from the sphere to the plane.

Curve $\mathrm{A}$-as computed neglecting space charge. Curve B-as estimated after space charge ball has reached plane.

the field near them so that photo electrons liberated in the region just ahead of a ball form new avalanches which are attracted to the ball, leaving a volume of high net positive space charge just in front of the sphere. The flow of electrons through the ball and back toward the sphere tends to reduce the net positive space-charge density in the path left behind the ball. Thus the ball of high positive space-charge density, which also acts as a center of high ionization and excitation activity, remains small but moves rapidly away from the sphere, creating the positive streamer trace on the film.

During this process the positive ions do not move appreciably, but remain essentially where they are produced, and the electrons move only short distances toward the sphere. The high speed of formation of the streamers is accounted for by the motion of the ball of high net positive space-charge density, which produces a momentary high voltage gradient at each point along the streamer path as it passes that point.

In the path left by the ball there is a high density of both positive ions and electrons, with a slight excess of positive ions. This net positive space charge changes the potential distribution between the sphere and plane, as indicated in figure 19 . Curve A is the potential computed from the applied voltage with space charge neglected. Curve B is an estimate of the potential after the ball has reached the plane. When the ball is at a given point $\mathrm{P}$, the potential follows curve B from the sphere to point $\mathrm{P}$ and curve $\mathrm{A}$ from point $\mathrm{P}$ to the plane. The discontinuity at point $\mathrm{P}$ is due to the high positive charge density in the ball, and it produces the high momentary gradient necessary to project the ball. The gradient as estimated from curve B is not high enough to produce ionization or excitation except in the region very near the sphere. The bright stems near the sphere (see figs. $4 \mathrm{E}^{\prime}$ and $14 \mathrm{~A}^{\prime}$ ) indicate ionization in this region, which probably persists

spacing of $20 \mathrm{~cm}$, and the steeply rising surge, the average number of streamers per shot was about 50 . 
until the additional space charge it produces decreases the gradient further. Thus the path left by the rapidly moving ball will not become a highly conducting plasma until some other mechanism for increasing ion density becomes active.

With the sphere negative, the electron avalanches formed in the region near the sphere initiate positive streamers which quickly propagate the short distance to the sphere. As the streamers approach the sphere, the high positive space-charge densities at their tips make the already extremely high gradients near the sphere even higher, and, largely because of these exceedingly high gradients, electrons are immediately released from the sphere.

The mechanism of electron release could be by any one or a combination of the following: (1) positive ion bombardment of the sphere, (2) high-energy photons impinging on the sphere, (3) field emission. In any event, more than sufficient electrons will be released to equalize the positive space-charge density, and a small volume or ball of high negative space charge will form near the sphere in line with each streamer. This is likely because of the high gradient near the sphere due to the applied voltage. Each small ball of high negative space-charge density can propagate away from the sphere by a mechanism nearly the same as for propagation of a positive ball, that is, not only by electron motion but largely because of the continuing generation of new electrons ahead of this volume. Here again, the ionizing and excitation activity is largely confined to this small ball of high space-charge density, which propagates rapidly toward the plane. Thus with the sphere negative, negative streamers develop from the sphere in much the same manner as positive streamers with the sphere positive. The experimental results indicate this to be true, as may be seen by comparing figures $4 \mathrm{~A}^{\prime}$ to $4 \mathrm{E}^{\prime}$ with figures $6 \mathrm{~A}^{\prime}$ to $6 \mathrm{D}^{\prime}$ (gap spacings too long to yield breakdown), and figures $12 \mathrm{~A}^{\prime}$ to $12 \mathrm{C}^{\prime}$ with figures $13 \mathrm{~A}^{\prime}$ to $13 \mathrm{C}^{\prime}$ (gap spacings giving breakdown but voltage chopped quickly after first discharge pip starts). The negative streamers leave numerous positive ions but a negative net space charge, which initially consists of electrons. The electrons, however, are quickly attached to oxygen molecules to form negative ions. Thus a potential distribution similar to that shown by curve $\mathrm{B}$ in figure 19 can persist for a short time and inhibit further ionization.

With the sphere negative, however, the initiation of a streamer requires a shorter gap spacing for a given applied voltage, and the velocity of propagation is less. To account for these experimental results, consider the development of an electron avalanche in the two cases: (1) sphere positive and (2) sphere negative. For the sphere positive, the electrons move in the direction of increasing gradient, because they travel toward the sphere or toward the ball of high positive space-charge density. For the sphere negative, the electrons move in the direction of decreasing gradient, because they travel away from the sphere or away from the ball of high negative space-charge density. Thus, the electron avalanches develop faster for the sphere positive, giving a higher velocity of streamer propagation. Also, the initiating avalanches can form positive streamers for lower applied gradients near the sphere with the sphere positive and thus produce streamer initiation for longer gap spacings.

The question now arises as to how much space charge would be required to suppress ionization, and whether the first discharge pip furnishes this amount. By applying Laplace's equation to the case of concentric spheres it can be shown that for a charge density in the space between the spheres, proportional to the reciprocal of the distance from the center, the gradient will be constant at all points between the spheres. By assuming such a charge distribution, and taking (1) the diameter of the inner sphere to be $1.6 \mathrm{~cm},(2)$ the diameter of the outer sphere to be $40 \mathrm{~cm}$, and (3) the potential difference between the speres as $145 \mathrm{kv}$, the total space charge required to give a uniform gradient was computed and found to be $3.4 \times 10^{-6}$ coulombs. The average first discharge pip for a 145-kv surge (sphere positive) and gap spacing of $20 \mathrm{~cm}$ was found experimentally to have a peak current of $19 \mathrm{amp}$ and a time to half value of $0.07 \mu \mathrm{sec}$. Integrating this current pulse to obtain the total charge supplied by it, gives 1.9 $\times 10^{-6}$ coulombs. This is about one-half the value computed for concentric spheres to give space charge yielding a uniform gradient. The factor one-half is about right to convert from the case of concentric spheres to sphere-plane electrodes.

The above computation is only intended to indicate that the space charge is of the correct order of magnitude to materially reduce the longitudinal voltage gradient in the region near the sphere. The assumptions used, which led to a constant gradient between sphere and plane, are not easily justified. It could be argued that the space charge per unit length of streamer (path left by the ball) would be constant. For concentric spheres this would give an average charge density proportional to the reciprocal of the square of the distance from the center. Computations based on such a charge distribution yield a gradient near the sphere actually higher than that obtained neglecting space charge. No definite statement as to exact charge distribution in the streamers can be made at this time. However, it should be readily possible for this space charge to alter the potential distribution as proposed in figure 19.

Another complication to be considered in such computations is that the space charge, just after formation of the streamers, would probably be mainly concentrated inside them. This would lead to a transverse gradient, i. e., perpendicular to the direction of the streamers. A rough computation of this transverse gradient can be made. The total number of streamers has already been estimated to be 50 (see footnote 5), and the streamer width as determined from measurements on figure $14 \mathrm{~A}^{\prime}$ is $3 \mathrm{~mm}$. By using these values and assuming the streamers to be cylinders with lengths equal to gap spacing, $L=20 \mathrm{~cm}$, the estimated total volume of all streamers is found to be $70.7 \mathrm{~cm}^{3}$, giving for $1.9 \times 10^{-6}$ coulomb total charge, an average charge density inside the 
streamers of $0.027 \times 10^{-6}$ coulomb $/ \mathrm{cm}^{3}$. The maximum voltage gradient at the surface of a streamer due to this charge was approximately computed and found to be about $18 \mathrm{kv} / \mathrm{cm}$, which is not sufficient to produce ionization. Thus, even when both longitudinal and transverse gradients are considered, the space charge introduced by the first discharge pip appears adequate to suppress further ionization.

\subsection{Second Discharge Rise and Breakdown}

For gap lengths sufficiently short or (in case voltage magnitude had been changed) for voltages sufficiently high, the first discharge pip is followed rather quickly by a second increase in current which, in general, rises at an increasing rate until breakdown occurs. As indicated in the experimental results, the mechanism of breakdown is different for the two sphere polarities.

\section{a. Sphere Positive}

With the sphere positive, a highly conducting channel starts to form at the surface of the sphere and grows toward the plane in a tree-like pattern with one or more main arms, each with many fingers which are fed by a multitude of fine filaments. This plasma channel acts as a conducting arm reaching out from the sphere, and as it extends itself toward the plane there is an ever-increasing gradient between the ends of the fingers and the plane. Thus, once started, it leads to complete breakdown unless the voltage is chopped.

The differences between streamers, which accompany the first discharge pip, and channels, which lead to breakdown, must be considered. They will be referred to merely as streamers and channels. Experimental evidence indicates the following differences: (1) Streamers propagate at a very high and probably nearly constant velocity of from 500 to $1,000 \mathrm{~cm} / \mu \mathrm{sec}$, while channels start to grow at a fairly low rate $(3 \mathrm{~cm} / \mu \mathrm{sec})$ and their rate of growth increases as they span more of the gap, being about $20 \mathrm{~cm} / \mu \mathrm{sec}$ at midgap; (2) streamers do not constitute a highly conducting plasma, but channels do; (3) streamers seem to follow along the original geometric lines of force of the electric field, but channels grow in a random zigzag course; (4) channels create a much more intense photographic record than streamers, suggestive of a much higher temperature and ion density in their core.

As already indicated, the streamers form quickly and leave behind a space-charge distribution which changes the original nonuniform field between sphere and plane to a more nearly uniform field. Thus immediately after the streamers have formed, the gradient at all points in the gap is below that required for ionization by collision, and if breakdown is to develop some other source of ionization must arise.

Although the streamers form almost instantly, the channels take enough time to develop so that the energy fed into their leading tip produces gas temperatures sufficiently high to yield a copious supply of ions. As the channel is formed it serves as a good conductor from its origin at the sphere to its leading end. Thus there will be a high gradient at the tip end of the channel, and filaments similar in their mechanism of formation to the initial discharge pip streamers will form at the tip end and feed more energy into the channel, causing it to propagate. The direction of channel formation at any instant depends upon the adjacent electric field strength and direction at that instant. The field configuration at any instant is fixed largely by the particular space charge distribution at that instant. Because the space charge distribution depends upon all previous ionization activity in the gap, it has random local irregularities and varies from instant to instant. This accounts for the random zigzag course of the channels.

After a channel has started it develops (as discussed above) until it bridges the gap, causing breakdown. Just how and why a channel starts is not self-evident. Because of the inhibiting space charge left by the streamers, it would appear that no further discharge could take place until this space charge had been at least partially cleared from the region near the sphere. This is, of course, true for longer gap spacings at which no breakdown occurs. As gap spacing is decreased a value is reached where breakdown occurs on some shots. In these cases the small bright stem of an initial streamer serves as the beginning of a bright channel. If the initial streamer pattern is such as to produce a sufficiently high gradient in a region near a bright stem, a channel will start to develop. Other streamer patterns may not produce a sufficiently high gradient. Thus the development of a channel is at least partly a matter of chance, the chances being greater for shorter gap spacings (assuming the same applied voltage surge). The fact that the channel does not continue to follow the same path as the streamer is readily accounted for by remembering that the gradient along the streamer (longitudinal gradient) tends to be uniform and fairly low because of space charge. Near the sphere the transverse gradient just outside a streamer should be much greater than the longitudinal gradient. Thus there should be more likelihood of a channel branching away from a streamer stem, as borne out by the experimental results. For the sphere positive (and provided not too great an overvoltage is applied), breakdown takes place along a zigzag: channel that has completely spanned the gap (see figs. $20 \mathrm{~A}$ and $21 \mathrm{~A}$ ).

\section{b. Sphere Negative}

With the sphere negative, breakdown is not initiated by a zigzag channel starting from the sphere. This would indicate that conditions in the region near the sphere are not propitious for channel development. One of these conditions is that the transverse gradient adjacent to the streamers be very high. This would hold for positive streamers because positive ions would diffuse into this region quite slowly. It would not hold for negative streamers, because electrons could quickly diffuse into lateral regions, forming negative ions and thereby reducing the gradient. The fact that zigzag 


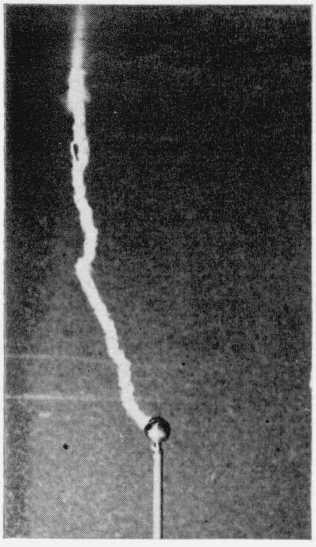

A

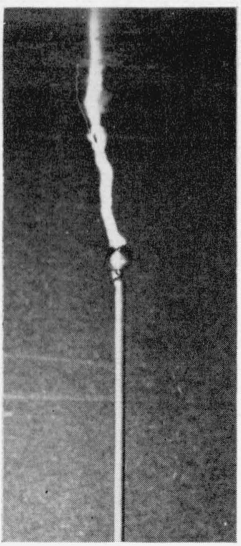

B

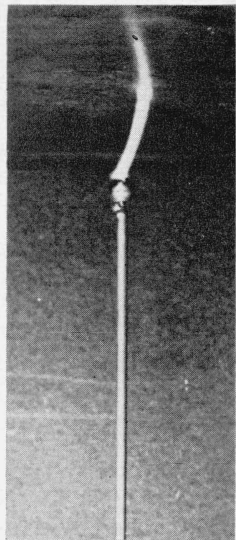

C
Figure 20. Spark discharge pictures (filter on camera). $0.07 \times 100,145$-kv surge, sphere positive. A, $L=20 \mathrm{~cm} ; \mathrm{B}, L=10 \mathrm{~cm} ; \mathrm{C}, L=5 \mathrm{~cm}$.

channels do not form from the sphere when it is negative helps to account for the observed result that, for the same voltage, a longer gap will break down when the sphere is positive than when it is negative.

It will be remembered that the appearance of negative streamers is quite similar to that of positive streamers for gap spacings longer than those at which breakdown may occur. The change in appearance with further decrease in gap spacing, at the same applied surge voltage, is presumably due to the higher gradients existing at the lower spacings. The most noteworthy change in appearance is the more intense photographic record, indicative of an increase in ionization activity. The increased brightness begins at the sphere and follows the streamers, going farther out into the gap the longer the voltage is left on (see figs. $17 \mathrm{~A}^{\prime}, 17 \mathrm{~B}^{\prime}$, and $17 \mathrm{C}^{\prime}$ ). This phenomenon requires a longer time to develop than the streamers, because when the voltage is chopped very quickly (see fig. 13) it does not occur. Thus it appears that the streamer itself develops into a straight channel as more energy is fed into it. Complete breakdown appears to occur in this way for a sufficiently short gap or sufficient overvoltage.

Another change in appearance is the addition of auxiliary streamers starting out nearly perpendicularly from the initial streamers and then curving down toward the sphere. These are probably positive streamers, initiated because of the high transverse gradient in the region between the initial streamers at a considerable distance from the sphere.

A third change in appearance is that the initial streamers acquire dots or small regions of greater ionization activity along their length. The spacing of these dots is random and some streamers show none. After the initial streamers have traversed the gap there is not sufficient voltage to maintain longitudinal ionizing gradients along the entire streamer. This is just barely true for the shorter gap spacings used with sphere negative, and if the gradient is not exactly uniform along the streamers these dots could be regions of high gradient. These

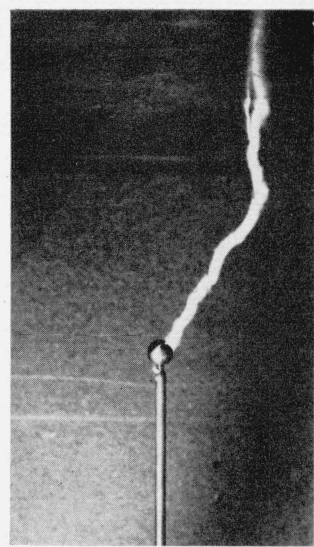

A

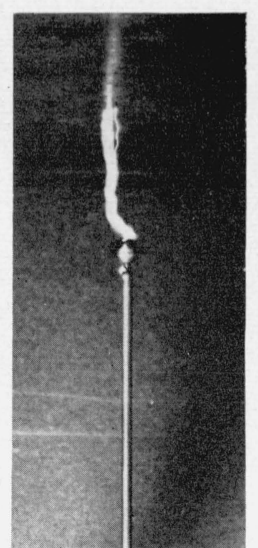

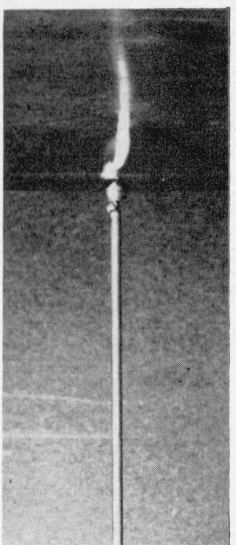

C
Figure 21. Spark discharge pictures (filter on camera). $1 \times 100,145$-kv surge, sphere positive. A, $L=15 \mathrm{~cm} ; \mathrm{B}, L=7.5 \mathrm{~cm} ; \mathrm{C}, L=3.8 \mathrm{~cm}$.

changes in appearance of negative streamers at gap settings leading to breakdown indicate that, for very short gap spacings or considerable overvoltage, breakdown would occur by a streamer developing into a channel as more energy is fed into it.

However, at gap spacings just short enough to give breakdown, there is another mechanism involved in breakdown. Plasma channels start from the plane and develop outward toward the sphere in random paths (see figs. $17 \mathrm{D}^{\prime}$ and $17 \mathrm{E}^{\prime}$ ) similar to the channels.which start from the sphere with sphere positive. These plane channels are probably initiated by high gradients at the plane created by the negative space charge of the initial streamers. They would start as positive streamers and quickly develop into channels, which follow a path perpendicular to the plane part way across the gap and develop further in a zigzag path by the same mechanism that produces zigzag channels from the positive sphere. Breakdown is then completed by these zigzag channels proceeding all the way to the sphere or meeting a channel developing from the sphere along the path of an initial negative streamer.

This explanation of breakdown with the sphere negative would predict that the mechanism of breakdown changes as gap spacing is reduced (or overvoltage increased). With very small overvoltage, breakdown would result from a zigzag channel developing from the plane. With high overvoltage, the breakdown channel would develop along the same path used by an initial streamer. This is shown to be true by the photographs of complete spark breakdowns for sphere negative (see figs. 22 and 23) taken with a dense filter at several different gap spacings. Similar photographs taken with the sphere positive (see figs. 20 and 21) indicate that here also, if gap spacing is sufficiently reduced, breakdown occurs by an initial streamer developing into a channel. These results suggest that the mechanism of breakdown in a uniform field gap is first a rapidly forming initial streamer, which because of the high gradients available quickly changes into a channel. This would account for 


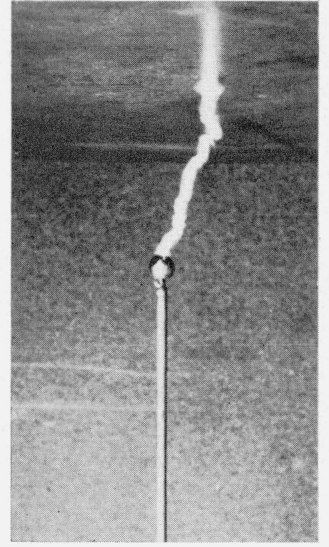

A

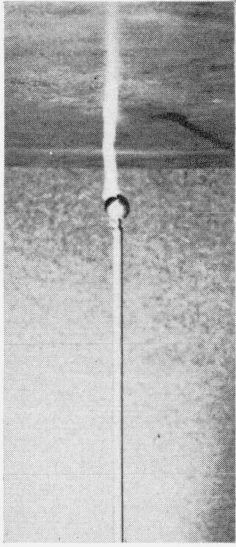

B

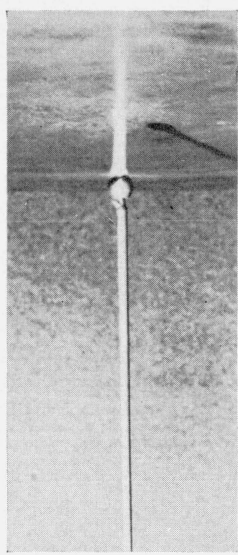

C
Figure 22. Spark discharge pictures (filter on camera). $0.07 \times 100,145 \mathrm{kv}$ surge, sphere negative. $\mathrm{A}, L=10 \mathrm{~cm} ; \mathrm{B}, L=6.5 \mathrm{~cm} ; \mathrm{C}$, $L=3.8 \mathrm{~cm}$.

the straight spark paths found for uniform field gaps [16].

\subsection{Effect of Rate of Voltage Rise}

The foregoing explanation of discharge mechanism in a nonuniform field gap has been based largely on data obtained when voltage is suddenly applied to the gap - that is, with a voltage rise time of 0.07 $\mu$ sec. Data obtained using a rise time of $1 \mu \mathrm{sec}$ were, in general, the same; but the magnitude of the first discharge pips and the size of the corresponding discharge patterns varied considerably from shot to shot. Because the first discharge pip and the ionization associated with it have such a short duration, they can happen on the rising front of a slowly rising voltage wave. The value of voltage that determines their magnitude is variable from shot to shot and is usually less than the peak value. After the initial discharge is formed, further discharge phenomena are inhibited by the space charge it leaves behind. Thus, unless gap spacing is such that complete breakdown occurs, no further discharges take place after the first discharge pip even though the applied surge voltage continues to rise. The magnitude of the first discharge phenomena depends on the exact value of voltage at the instant of discharge initiation. Consequently, any scattering in "time to first discharge pip" results in a considerable variation in current magnitude and in corresponding photographic records.

Experimental results showed that the later the first discharge pip occurred on the rising front of the voltage wave (that is, the higher the voltage at instant of occurrence), the larger the magnitude of first discharge pip and streamer length. The transition from initial discharge to breakdown is probably very nearly the same for a $1-\mu$ sec front voltage as for the faster voltage front, as may be seen for the sphere positive by comparing the records shown in figure 16 with those in figure 14 . With the sphere negative the initial discharges do not appear to extend as far into the gap (see fig. 7), and there might

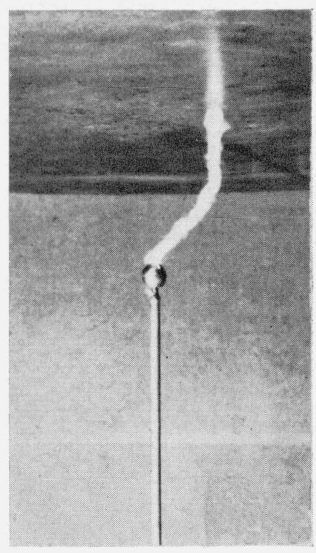

A

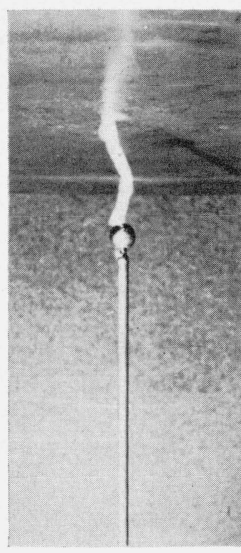

B

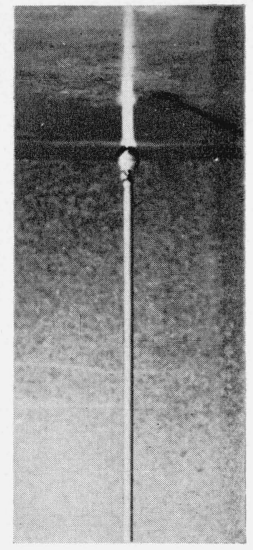

C
Figure 23. Spark discharge pictures (filter on camera).

$1 \times 100,145$-kv surge, sphere negative. A, $L=9 \mathrm{~cm} ; \mathrm{B},{ }^{\circ} L=6.5 \mathrm{~cm} ; \mathrm{C}, L=3.8 \mathrm{~cm}$.

be a question as to whether plate stems and channels can form. However, photographic records (fig. 18) using chopped voltages show that they do form, and breakdown occurs in much the same manner as for the steeply rising voltage. This can be accounted for when one realizes that the streamers may extend farther than the photographic records show. At the ends of these streamers a large supply of electrons is produced during the first discharge pip, and there is an interval of nearly $1 \mu$ sec before initiation of the plate stems (as seen in fig. 18). During this interval, these electrons can drift toward the plane and, as a negative space charge, cause the high gradients necessary there to initiate positive streamers and channels.

The experimental curves shown in figure 10 indicate another difference in breakdown characteristic depending upon the rate of voltage rise. These curves show that for a steeply rising surge, breakdown is more apt to occur at the greater gap spacings than with a slowly rising surge. As already mentioned, the particular pattern of the initial discharge streamers, which varies from shot to shot, determines whether or not breakdown will occur. In general, probably, the greater the magnitude (i. e., total coulombs) of the first discharge pip, the greater the chance for breakdown to follow. The first discharge pip is of higher magnitude on the average for a steeply rising surge, and thus breakdown is more likely to develop at greater gap spacings.

\section{Summary}

1. When a surge voltage of sufficient magnitude and duration is applied to a sphere-plane gap (nonuniform field) the prebreakdown discharge phenomena are divided into two phases: (a) the initial streamers which produce the first discharge pip and (b) the somewhat later development of a good conducting channel which causes a second rise in discharge current. This second current rise increases exponentially to breakdown unless the applied voltage is chopped. 
2. The statistical nature of the experimental results is demonstrated by summaries of the following measured quantities: (a) time delay from start of voltage surge to first discharge pip, (b) peak current value of first discharge pip, (c) percentage of complete breakdowns for a given gap spacing, and (d) time delay from first discharge pip to breakdown. Before voltage is applied the instantaneous ion pattern near the sphere is changing continuously, thus accounting for the variations in quantities (a) and (b) on successive applications of the surge. The random nature of quantities (c) and (d) is accounted for by variations in the first discharge streamer magnitude and pattern on repeated surge applications.

3. The initial streamers propagate from the sphere toward the plane along lines of force of the applied electric field at very high velocities, $500 \mathrm{~cm} / \mu \mathrm{sec}$ for the sphere negative and $800 \mathrm{~cm} / \mu \mathrm{sec}$ for the sphere positive. During the initial discharge the surge of current that flows to the sphere rises to a peak value of 1 to $30 \mathrm{amp}$ (depending on gap spacing and sphere polarity) in about $0.008 \mu \mathrm{sec}$ and then decreases approximately exponentially to zero, reaching half value in about $0.08 \mu \mathrm{sec}$. For gap spacings at which breakdown is likely to occur, the initial streamers bridge the entire gap from sphere to plane without causing immediate breakdown. The formation of the initial streamers produces a space-charge distribution in the gap, which apparently inhibits further ionization unless conditions are such that breakdown can occur.

4. If complete gap breakdown is to take place, a second rise in current signifies the development of a conducting channel between the electrodes. With the sphere positive this channel starts to form near the surface of the sphere and follows a zigzag path to the plane. With the sphere negative the conducting channel starts out perpendicular to the plane, but after a short distance changes its course and makes a zigzag path to the sphere. Breakdown is

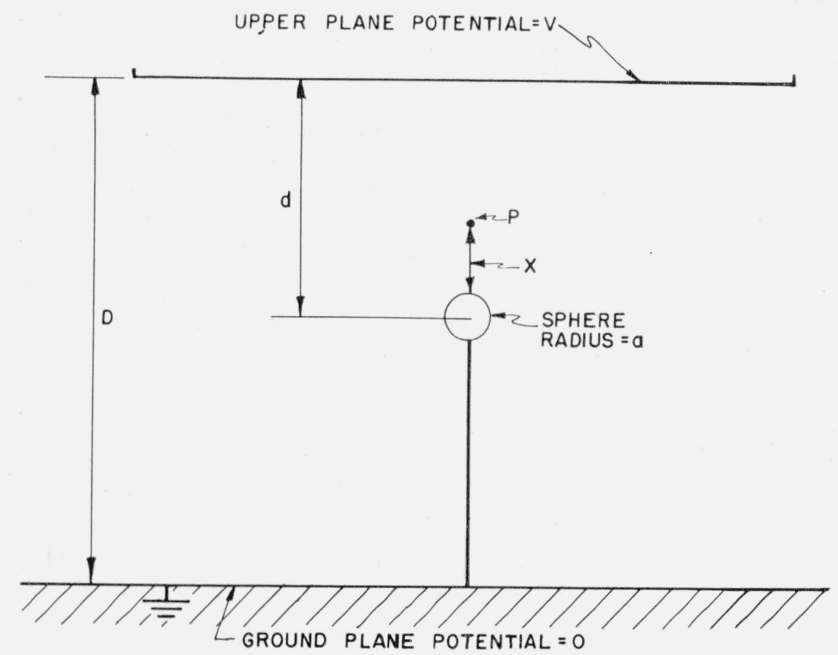

Figure 24. Schematic diagram of sphere-plane electrodes as used to obtain experimental results.

In Peek's formula, eq (1), $\delta=d-a$ and $y=d-a-x$. completed when this highly conducting channel completely spans the gap.

5. For gap spacings much shorter than those first causing breakdown, i. e., for considerable overvoltage, the gap breakdown occurs by the development of one of the initial streamers into a conducting channel. The breakdown path is then not zigzag but more nearly straight and seemingly follows a "line of force" of the geometric field. It is suggested that this is the normal type of breakdown for uniform field gaps.

\section{Appendix. Computation of Voltage Gra- dient Between a Sphere and a Plane}

For an infinite plane and sphere isolated in space and maintained at a constant potential difference, the gradient along the perpendicular from the plane to the center of the sphere can be computed by a formula given by Peek [17]. In this formula:

$V=$ Potential difference between sphere and plane.

$a=$ radius of the sphere.

$\delta=$ the distance from the plane to the surface of the sphere along the perpendicular from the plane to the center of the sphere.

$y=$ the distance from the plane to the point at which gradient is to be determined along the perpendicular from the plane to the center of the sphere.

$p=1+\delta / a$

$f=\frac{1}{4}\left[(2 p-1)+\sqrt{\left.(2 p-1)^{2}+8\right]}\right.$

$E_{y}=$ gradient along the perpendicular from the plane to the center of the sphere at a distance $y$ from the plane. $E_{y}$ is then given in volts per centimeter if dimensions are in centimeters and $V$ is in volts by the equation

$$
E_{y}=V \frac{2 \delta\left[\delta^{2}(f+1)+y^{2}(f-1)\right]}{\left[\delta^{2}(f+1)-y^{2}(f-1)\right]^{2}} .
$$

Results obtained using eq (1) would be correct if the plane were at ground potential, located on the floor, and the sphere at the high potential located above the plane, provided the effect of the lead connecting the sphere to the high potential could be neglected. In the present experiments these conditions were not fulfilled and Peek's formula was not considered to be a sufficiently close approximation.

Figure 24 shows the arrangement of (1) ground plane at potential zero, (2) high voltage plane at potential $V$, and (3) sphere at potential zero as used in the present experiments. By neglecting the effect of the tube supporting the sphere, a system of charges $\mathrm{Q}_{1}, \mathrm{Q}_{1}^{\prime}, \mathrm{Q}_{1}^{\prime \prime}, \mathrm{Q}_{2}^{\prime}$, and $\mathrm{Q}_{2}^{\prime \prime}$ can be chosen to make the potential very nearly zero at every point on the surface of the sphere. The magnitude and position of $\mathrm{Q}_{1}$ is chosen so that it, together with the surface charges on the two planes, makes the potential zero everywhere on the surface of the sphere. Charges $Q_{1}^{\prime}$ and $Q_{1}^{\prime \prime}$ are image charges for $Q_{1}$ which 
keep the potentials of the two planes at their proper values. Charges $\mathrm{Q}_{2}^{\prime}$ and $\mathrm{Q}_{2}^{\prime \prime}$ are image charges inside the sphere of $\mathrm{Q}_{1}^{\prime}$ and $\mathrm{Q}_{1}^{\prime \prime}$ to keep the sphere at zero potential. Further image charges were neglected. Using this system of charges and making some approximations based on $a$ being much smaller than $d$ or $D$, the following formula for $E_{x}$, the gradient at the distance $x$ from the surface of the sphere along the perpendicular from the high voltage plane to the center of the sphere, was derived:

$$
\begin{aligned}
E_{x}= & V\left\{\frac{1}{D}+\frac{D-d}{D} a\left[\frac{1}{\left(a-\frac{a^{2}}{D-d}+x\right)^{2}}+\frac{1}{(2 d-a-x)^{2}}\right.\right. \\
& -\frac{1}{[2(D-d)+a+x]^{2}}+\frac{a}{2 d\left(a-\frac{a^{2}}{2 d}+x\right)^{2}} \\
& \left.\left.+\frac{a}{2(D-d)\left[a+\frac{a^{2}}{2(D-d)}+x\right]^{2}}\right]\right\}
\end{aligned}
$$

In this equation the effect of the mounting tube for the sphere was neglected, which is probably justified if its diameter is small. To determine approximately how well this holds, a more complicated computation was carried out for the specific case of $d=10 a$, $D=40 a$, and radius of the mounting tube $=a / 4$. Charges along the axis of the mounting tube were considered with their images in addition to the charges used in the derivation of eq (2). They were chosen to make the potential at the surface of the mounting tube nearly zero at all points. The results of this computation for the one specific case mentioned are compared with those obtained by eq (1) and (2) in the following table:

\begin{tabular}{|c|c|c|c|}
\hline \hline & $\begin{array}{c}\text { Mounting } \\
\text { tube con- } \\
\text { sidered }\end{array}$ & \multicolumn{2}{|c|}{ Equation } \\
\cline { 3 - 4 } & (2) & (1) \\
\cline { 2 - 4 } $\begin{array}{c}\text { Gradient at surface of the sphere } \\
\text { in volts per centimeter.....- }\end{array}$ & $0.8715 \times V$ & $0.884 \times V$ & $1.061 \times V$ \\
\hline
\end{tabular}

The value obtained using eq (2) in which the mounting tube was neglected is nearly the same as that obtained when mounting tube was considered. Both values are somewhat lower than that obtained using eq (1), Peek's formula. Equation (2) was considered to be a sufficiently close approximation and for the actual dimensions used in the experimental work and for $145 \mathrm{kv}$ applied to the plane the values of gradient at various distances from the sphere are shown in figure 25 for gap spacings of 10 , $20,30,40$, and $50 \mathrm{~cm}$.

Washington, December 28, 1955.

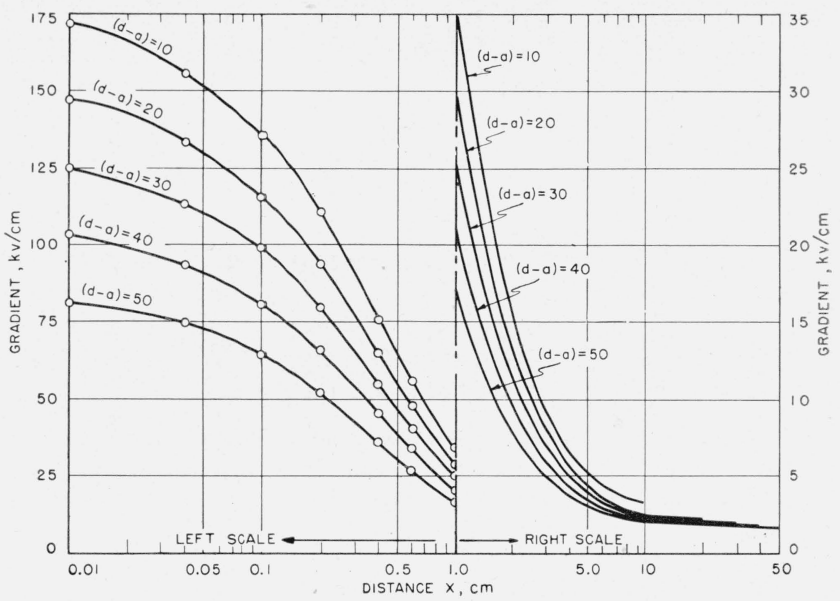

FIgURE 25. Curves of voltage gradient as computed from eq (2) plotted against distance, $x$, from the surface of the sphere (see figure 24).

$V=145 \mathrm{kv}, D=86.4 \mathrm{~cm}, a=0.794 \mathrm{~cm}$.

\section{References}

[1] Leonard B. Loeb, Basic processes of gaseous electronics, chs. VIII and IX (Univ. of California Press, 1955). Additional references at the end of each chapter in this book.

[2] F. A. Maxfield and R. R. Benedict, Theory of gaseous conduction and electronics, page 270 (McGraw-Hill Book Co., Inc., New York, N. Y., 1941).

[3] F. Llewellyn Jones and A. B. Parker, Electrical breakdown of gases - I. Spark mechanism in air, Proc. Roy. Soc. London [A] 213, 185 (1952).

[4] J. Dutton, S. C. Hayden, and F. Llewellyn Jones, Formative time lags in the electrical breakdown of gases, Brit. J. Appl. Phys. 4, 170 (1953).

[5] Leonard B. Loeb and John M. Meek, The mechanism of the electric spark (Stanford University Press, 1941).

[6] M. A. Biondi, Microwave gas discharge, Elec. Eng. 69, 806 (1950).

[7] W. P. Allis and S. C. Brown, High frequency electrical breakdown of gases, Phys. Rev. 87, 419 (1952).

[8] Sanborn C. Brown, High-frequency gas-discharge breakdown, Proc. IRE 39, 1493 (1951).

[9] Harald Norinder and Oskars Salka, Mechanism of positive spark discharges with long gaps in air at atmospheric pressure, Arkiv Fysik 3, No. 19, 347 (1951).

[10] Harald Norinder and Oskars Salka, Mechanism of longgap negative spark discharges in air at atmospheric pressure, Arkiv Fysik 5, No. 24, 493 (1952).

111] Harald Norinder and Oskars Salka, Screens in long discharge gaps, Arkiv Fysik 6, No. 17, 151 (1953).

[12] J. H. Hagenguth, A. F. Rohlfs, and W. J. Degnan, Sixty-cycle and impulse sparkover of large gap spacings, Trans. AIEE $\boldsymbol{\gamma 1}, 455$ (1952).

[13] R. F. Saxe and J. M. Meek, The initiation mechanism of long sparks in point-plane gaps, Inst. Elec. Engr. London 102C, 221 (1955).

[14] John H. Park, A fifty-fold momentary beam intensification for a high-voltage cold-cathode oscillograph, J. Research NBS 47, 87 (1951) R P2231.

[15] G. H. Johnson, An impulse generator circuit for choppedwave tests on transformers, Trans. AIEE $\mathbf{7 \% ,} 839$ (1953)

[16] F. M. Bruce, High-voltage spark discharge, Endeavour XIII, No. 50, 61 (Apr. 1954).

[17] F. W. Peek, Dielectric phenomena in high-voltage engineering, p. 30 (McGraw-Hill Book Co., New York N. Y., 1915). 